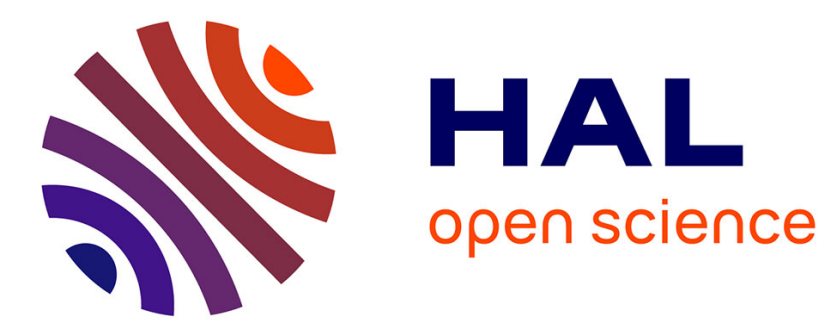

\title{
An approximate Roe-type Riemann solver for a class of realizable second order closures
}

\author{
Gilles Brun, Jean-Marc Hérard, Denis Jeandel, Markus Uhlmann
}

\section{To cite this version:}

Gilles Brun, Jean-Marc Hérard, Denis Jeandel, Markus Uhlmann. An approximate Roe-type Riemann solver for a class of realizable second order closures. International Journal of Computational Fluid Dynamics, 2000, 13 (3), pp.223-249. 10.1080/10618560008940900 . hal-01580047

\section{HAL Id: hal-01580047 \\ https://hal.science/hal-01580047}

Submitted on 23 Dec 2019

HAL is a multi-disciplinary open access archive for the deposit and dissemination of scientific research documents, whether they are published or not. The documents may come from teaching and research institutions in France or abroad, or from public or private research centers.
L'archive ouverte pluridisciplinaire HAL, est destinée au dépôt et à la diffusion de documents scientifiques de niveau recherche, publiés ou non, émanant des établissements d'enseignement et de recherche français ou étrangers, des laboratoires publics ou privés. 


\title{
An Approximate Roe-type Riemann Solver for a Class of Realizable Second Order Closures
}

\author{
G. Brun \\ Société METRAFLU \\ 64, chemin des Mouilles \\ 69134 Ecully Cedex \\ France \\ J.-M. Hérard \\ DER - LNH, Electricité de France \\ 6, quai Watier \\ 78400 Chatou \\ France \\ jean-marc.herard@der.edf.fr \\ D. Jeandel, M. Uhlmann* \\ LMFA, Ecole Centrale de Lyon \\ 36, avenue Guy de Collongue \\ 69131 Ecully \\ France
}

July 19, 1999

\begin{abstract}
A realizable, objective second-moment turbulence closure, allowing for an entropy caracterisation, is analyzed with respect to its convective subset. The distinct characteristic wave system of these equations in non-conservation form is exposed. An approximate solution to the associated one-dimensional Riemann problem is constructed making use of approximate jump conditions obtained by assuming a linear path across shock waves. A numerical integration method based on a new approximate Riemann solver (flux-difference-splitting) is proposed for use in conjunction with either unstructured or structured grids. Test calculations of quasi one-dimensional flow cases demonstrate the feasibility of the current technique even where Euler-based approaches fail.
\end{abstract}

\section{Introduction}

Second order modelling of the Reynolds stress tensor has received growing attention over the past two decades. This particular closure technique has considerably matured so that today, Reynolds stress transport models are in use over a broad range of turbulent flow types in engineering applications $[1,2,3]$.

In the compressible flow regime, one has to deal with wavelike phenomena including discontinuities (e.g. shock waves) due to the hyperbolic character of the convective subset of the governing equations. These specific flow features constitute a major challenge to any type of numerical simulation. In the past, characteristics based methods have proven very useful notably for solving the equations of gas dynamics $[4,5]$. The underlying idea of this class of numerical techniques is to incorporate as much as possible of the physics of the analytical problem into the discrete treatment. An important building block in this respect has been the one-dimensional Riemann problem which

* Now at School of Aeronautics, U. Politécnica Madrid 
exhibits the essential features of characteristic wave propagation in the presence of discontinuities. In the case of the well-known Euler equations, an analytical solution to the Riemann problem can be found making use of the conservation form of the system (Rankine-Hugoniot relations). When dealing with Reynolds averaged transport equations in conjunction with a second order closure, the hyperbolic subset is not in conservation form due mainly to production terms (a similar situation arises in two-phase flows [6]). The fact that no classical analytical solution can be found for this system in the presence of discontinuities hinders the construction of a numerical method.

In past applications of second-moment closures to compressible flow problems this difficulty has sometimes been circumvented resorting to the gas dynamics case as a model for physical propagation properties thus effectively neglecting the influence of turbulence on the characteristic wave system $[7,8]$. However, recent work suggests that this simplified approach violates thermodynamic realizability properties [9] and can lead to non-physical or even unstable numerical solutions [10, 11].

An analysis of the complete hyperbolic subset in the framework of two-equation turbulence models ( $k-\varepsilon$ type closures) has been carried out by Louis [12] and Forestier et al. [13]. These authors made use of the assumption of a linear path of a particular set of dependent variables across shock waves in order to derive an approximate analytical solution of the Riemann problem, eventually leading to an appropriate numerical method. The aim of the present article is to extend that technique upon systems issuing from second-moment closures. However, the tensorial character of the Reynolds stress further complicates the task through supplementary constraints of realizability which are strongly tied to the hyperbolicity of the underlying system. Before even directing ones attention on the hyperbolic subset, it is thus important to assure that the closed set of (modelled) equations meets the entire criteria that can be set forth from the exact equations of motion.

The outline of the present article is as follows. In section one we present a class of secondmoment closures that exhibit the desired realizability properties and allows for a clear entropy caracterisation. We then focus on the analysis of the associated hyperbolic subset which is described in section two. Approximate jump conditions are proposed for the non-conservative system. We then go on to construct the solution to the one-dimensional Riemann problem, applying the entropy inequality and restricting to weak shocks. The presented solution fulfills realizability requirements. These results enable us to propose in section 4 a simple but efficient way to compute time-dependent solutions including rarefaction waves, shocks and contact discontinuities, either using structured or unstructured meshes. The fourth section is devoted to the presentation of sample computational results of turbulent shock tube experiments, which confirm the capabilities of the scheme, even for high values of the turbulent Mach number.

\section{The second moment closure}

\subsection{Governing equations}

In this paper, we resort to Favre averaging of the instanteneous Navier-Stokes equations [14] while neglecting fluctuations of "molecular" quantities (viscosity and heat conductivity). Two supplementary hypotheses are applied: the turbulent mass flux and temperature-density correlations are neglected when appearing in conjunction with viscosity; the turbulent heat flux is expressed by a gradient transport type model for simplicity. The resulting set of equations can then be written 
as follows (for more details on the derivation we refer to $[15,16,11])$ :

$$
\begin{array}{lll}
(\rho)_{, t}+\left(\rho U_{j}\right)_{, j} & =0 \\
\left(\rho U_{i}\right)_{, t}+\left(\rho U_{i} U_{j}+\delta_{i j} p+\rho R_{i j}\right)_{, j} & =\left(\Sigma_{i j}\right)_{, j} \\
(\rho E)_{, t}+\left(\rho E U_{j}+U_{i}\left(p \delta_{i j}+\rho R_{i j}\right)\right)_{, j} & =\left(U_{i} \Sigma_{i j}+\sigma_{E}\left(\frac{p}{\rho}\right)_{, j}\right)_{, j}+\frac{1}{2} \mathcal{T}_{k k j, j}-\left(\overline{u_{j}^{\prime \prime}} p\right)_{, j} \\
\left(\rho R_{i j}\right)_{, t}+\left(\rho R_{i j} U_{k}\right)_{, k}+\rho R_{i k} U_{j, k}+\rho R_{j k} U_{i_{, k}} & =\Phi_{i j}-\frac{2}{3} \delta_{i j} \rho \varepsilon+\mathcal{T}_{i j k_{, k}}-\overline{u_{i}^{\prime \prime}} p_{, j}-\overline{u_{j}^{\prime \prime}} p_{, i}
\end{array}
$$

Tilde, overbar and primes have been dropped in (1) except where needed for clarity. $\rho$ stands for the mean density, $\mathbf{U}$ is the density weighted mean velocity vector, $\mathbf{R}$ the Reynolds stress tensor $R_{i j}=\widehat{u_{i}^{\prime \prime} u_{j}^{\prime \prime}}, E$ the mean specific total energy and $p$ the mean pressure which can be expressed via the ideal gas law (with $\gamma$ being the ratio of specific heats), viz.:

$$
p=(\gamma-1)\left(\rho E-\frac{1}{2} \rho U_{k} U_{k}-\frac{1}{2} \rho R_{k k}\right) .
$$

The mean viscous stress $\boldsymbol{\Sigma}$ is defined as:

$$
\Sigma_{i j}=\mu\left(U_{i, j}+U_{j, i}-\frac{2}{3} \delta_{i j} U_{k, k}\right),
$$

where $\mu$ is the dynamic molecular viscosity. $\sigma_{E}$ is the (positive) coefficient of total heat flux (including a turbulent and a mean contribution); $\mathcal{T}_{i j k}$ regroups all turbulent transport terms of the Reynolds stress; $\overline{u_{i}^{\prime \prime}}$ represents the turbulent mass flux, corresponding to the difference between density weighted and Reynolds averaged mean velocity. $\boldsymbol{\Phi}$ stands for the sum of the pressure-strain correlation and the non-isotropic part of the dissipation tensor [17]; $\varepsilon$ is the turbulent mechanical dissipation rate which can be obtained by a standard model equation (e.g. [18]).

\subsection{Realizability properties}

We now recall the basic conditions to be fulfilled a priori by our second moment closure. Admissible states for the Reynolds stress tensor are such that the following inequality holds for any unit vector $\mathbf{n}$ in $\mathbb{R}^{3}$ :

$$
\mathbf{n}^{t} \cdot \mathbf{R}(\mathbf{x}, t) \cdot \mathbf{n} \geq 0,
$$

which expresses that fluctuating velocities must be real. As a direct consequence of (4) one obtains a set of constraints:

$$
f \geq 0 \quad\left(f=\left\{\delta_{1}^{i}, \delta_{2}^{i}, \delta_{3}\right\} \quad i=1,3\right),
$$

where $f$ designates any one amongst the fundamental minors of the Reynolds stress:

$$
\delta_{1}^{\alpha}=R_{\alpha \alpha}, \quad \delta_{2}^{\alpha}=R_{\beta \beta} R_{\gamma \gamma}-R_{\beta \gamma}^{2}, \quad \delta_{3}=\operatorname{det}(\mathbf{R}) .
$$

As a further criterion, mean density and pressure need to be positive, viz:

$$
\begin{aligned}
& \rho(\mathbf{x}, t) \geq 0 \\
& p(\mathbf{x}, t) \geq 0 .
\end{aligned}
$$

We thus define:

A closed set of equations that assures solutions complying with inequalities (5), (7) and (8) is called weakly realizable.

Considering the exact limiting behaviour of the Reynolds stress tensor, one can go further and formulate the following requirement of strong realizability $[17,19,20,21,22,23,24,25]$ :

$$
f \geq 0 \quad \cup \quad f=0 \quad \Rightarrow \quad\left(\mathrm{d}_{t}(f)=0 \quad \cup \quad \mathrm{d}_{t t}(f) \geq 0\right),
$$


with $f=\left\{\delta_{1}^{i}, \delta_{2}^{i}, \delta_{3}\right\}$ and $\mathrm{d}_{t}=\partial_{t}()+U_{k}()_{, k}$ the material derivative and $\mathrm{d}_{t t} f=\mathrm{d}_{t}\left(\mathrm{~d}_{t} f\right)$.

For the sake of completeness, we call Reynolds stress closures that do not allow the solution to approach the limiting state, i.e. such systems that verify

$$
f \geq 0 \quad \cup \quad f=0 \quad \Rightarrow \quad \mathrm{d}_{t}(f) \geq 0,
$$

over-realizable.

Finally, it has been shown by Speziale [26] that the exact individual terms of the Reynolds stress transport equation (with the exception of production) are invariant under arbitrary accelerations of the frame of reference. Respective models should thus be formultated in a manner corresponding to this so-called objectivity requirement.

In the absence of a full proof of realizability for non-gaussian closures [22] we restrict our scope in the following to gaussian closures $\left(\mathcal{T}_{i j k}=0\right)$.

Lumley [17] proposed a model for the slow part of the pressure-strain correlation $\boldsymbol{\Phi}$ which is in agreement with previous constraints (weak realizability, strong realizability, objectivity) and allows for a return-to-isotropy mechanism [24]. It must be pointed out that more recently strongly realizable models for the rapid part of pressure-strain have been put forth [20, 21] which however do not fulfil the objectivity requirement. We thus focus herein on Lumley's proposal which reads

$$
\Phi=-\alpha\left(I, I I, I I I, R e_{l}\right) \cdot \rho \varepsilon \cdot\left(\frac{R_{i j}}{R_{k k}}-\frac{1}{3} \delta_{i j}\right),
$$

where $\alpha\left(I, I I, I I I, R e_{l}\right)$ is a dimensionless function of the three invariants $I, I I, I I I$ of the Reynolds stress tensor and a turbulent Reynolds number $R e_{l}$.

Moreover, we refrain from the practice of including an explicit model for the trace of pressurestrain, the so-called pressure-dilatation correlation, since algebraic expressions that have been used in the past $[27,28,29,30,31]$ are in conflict with the constraints of strong realizability (cf. [11]).

The turbulent mass flux $\overline{u_{i}^{\prime \prime}}$ is assumed to be modelled by a generalized gradient transport expression as proposed by Zeman [32] and Ristorcelli [33]:

$$
\overline{u_{i}^{\prime \prime}}=\chi \frac{R_{i l}}{\rho} \rho_{, l},
$$

where $\chi$ designates a characteristic time scale of the energetic eddies.

\subsection{Entropy inequality}

The system of equations (1) in conjunction with our modelling assumptions of the preceding paragraph enables us to derive an entropy inequality analogous to the case of the instantaneous Navier-Stokes equations (cf. e.g. [34]). Introducing a vector $\mathbf{W}^{3 D}$ of "pseudo-conservative" variables

$$
\mathbf{W}^{3 D}=\left(\rho, \rho U, \rho V, \rho W, \rho E, \rho R_{11}, \rho R_{22}, \rho R_{33}, \rho R_{12}, \rho R_{13}, \rho R_{23}\right)^{t},
$$

and the entropy function

$$
\eta\left(\mathbf{W}^{3 D}\right)=-\rho \log \left(p / \rho^{\gamma}\right),
$$

we obtain:

Proposition 1: Regular solutions of the set (1) are such that

$$
\eta, t+\nabla \cdot f_{\eta}^{n v}\left(\mathbf{W}^{3 D}\right)+\nabla \cdot f_{\eta}^{v}\left(\mathbf{W}^{3 \mathbf{D}}, \nabla \mathbf{W}^{3 D}\right)=S_{\eta}\left(\mathbf{W}^{3 D}, \nabla \mathbf{W}^{3 D}\right) \leq 0,
$$

where the fluxes are defined as $(T=p / \rho)$ :

$$
\begin{aligned}
f_{\eta}^{n v} & =\mathbf{U} \cdot \eta, \\
f_{\eta}^{v} & =-\left(\chi R_{k l} \rho_{, l}(\gamma-1)\right)_{, k}+\left((\gamma-1) \sigma_{E} \frac{T_{, k}}{T}\right)_{, k},
\end{aligned}
$$


and the source term may be written as:

$$
S_{\eta}=-\frac{\gamma-1}{T}\left(\frac{\sigma_{E}}{T} T_{, i}^{2}+\rho \varepsilon+\Sigma_{i j} \cdot U_{i, j}\right)-(\gamma-1) \chi \frac{R_{i j}}{\rho} \rho_{, i} \rho_{, j} .
$$

This clear inequality of the full set of model equations becomes useful in the inviscid limit where the following property holds across a discontinuity of speed $\sigma$ :

$$
-\sigma[\eta]+\left[f_{\eta}^{n v}\right] \leq 0
$$

With the help of inequality (19) the physically correct jump condition in the case of a shock wave can be selected amongst mathematically possible candidates. This is a prerequisite for the unique solution of the Riemann problem in section 3.3.

\section{The convective subset}

\subsection{Introduction}

The convective subset of our second-moment closure is obtained by setting all viscosity and conductivity related terms to zero in equations (1) and retaining only first order differential expressions. Alternatively, one can start off with the inviscid instantaneous equations (Euler) and carry out the statistical treatment. After introducing the above mentioned modelling assumptions the result is the following system:

$$
\begin{array}{llll}
(\rho)_{, t} & +\left(\rho U_{j}\right)_{, j} & = & 0 \\
\left(\rho U_{i}\right)_{, t} & +\left(\rho U_{i} U_{j}+\delta_{i j} p+\rho R_{i j}\right)_{, j} & = & 0 \\
(\rho E)_{, t}+\left(\rho E U_{j}+U_{i}\left(p \delta_{i j}+\rho R_{i j}\right)\right)_{, j} & = & 0 \\
\left(\rho R_{i j}\right)_{, t}+\left(\rho R_{i j} U_{k}\right)_{, k} & = & -\rho R_{i k} U_{j_{, k}}-\rho R_{j k} U_{i_{, k}}
\end{array}
$$

Equations (20) cannot be put into conservation form due to the presence of the turbulence production term on the right hand side. From this fact stems the main difficulty in analyzing and solving the equations issuing from second-moment closure: classic results from the theory of hyperbolic systems of conservation laws (cf. e.g. [35, 34]) cannot be simply "applied" to this non-conservative system.

In the following we restrict our investigation to statistically two-dimensional turbulent flow (not to be confused with the extreme state of two-dimensional turbulence) in order to keep algebraic manipulations tractable. An extension to three dimensions should be straightforward but cumbersome. We thus assume that

$$
R_{13}=R_{23}=W=0,
$$

and

$$
\phi, 3=0
$$

whatever $\phi$ stands for. With the new vector of state variables

$$
\mathbf{W}=\left(\rho, \rho U, \rho V, \rho E, \rho R_{11}, \rho R_{22}, \rho R_{33}, \rho R_{12}\right)^{t},
$$

the convection-production subset (20) now reads

$$
\mathbf{W}_{, t}+\left(\mathbf{F}_{i}(\mathbf{W})\right)_{, i}=\mathbf{H}(\mathbf{W}, \nabla \mathbf{W}) \quad i=1,2
$$

where $\mathbf{F}_{i}(\mathbf{W})$ are the convective fluxes and $\mathbf{H}(\mathbf{W}, \nabla \mathbf{W})$ the production term. We can assemble a system matrix $\mathbf{A}_{i}(\mathbf{W})$

$$
\mathbf{A}_{i}(\mathbf{W})=\frac{\partial \mathbf{F}_{i}(\mathbf{W})}{\partial \mathbf{W}}+\mathbf{C}_{i}^{n c}(\mathbf{W}) \quad i=1,2
$$


where

$$
\mathbf{C}_{i}^{n c}(\mathbf{W}) \cdot \mathbf{W}_{, i}=-\mathbf{H}(\mathbf{W}, \nabla \mathbf{W}),
$$

so that equation (24) takes on the following familiar form:

$$
\mathbf{W}_{, t}+\mathbf{A}_{i} \cdot \mathbf{W}_{, i}=0 .
$$

\subsection{Hyperbolicity}

Once more resorting to a unit vector $\mathbf{n}$ we define

$$
U_{n}=\mathbf{U}^{t}(\mathbf{x}, t) \mathbf{n}, \quad R_{n n}=\mathbf{n}^{t} \mathbf{R}(\mathbf{x}, t) \mathbf{n} .
$$

Since the system of equations is invariant under rotation, it suffices to investigate its characteristics in a single arbitrary direction $\mathbf{n}$. We obtain with respect to the eigenvalues:

Proposition 2: The convective subset (27) is a non strictly hyperbolic system of equations if conditions (5), (7) and (8) of weak realizability hold. Eigenvalues are (in ascending order):

$$
\begin{aligned}
& \lambda_{1}=U_{n}-c_{1} \\
& \lambda_{2}=U_{n}-c_{2} \\
& \lambda_{3}=\lambda_{4}=\lambda_{5}=\lambda_{6}=U_{n} \\
& \lambda_{7}=U_{n}+c_{2} \\
& \lambda_{8}=U_{n}+c_{1},
\end{aligned}
$$

with

$$
\begin{aligned}
& c_{1}=\sqrt{\gamma p / \rho+3 R_{n n}} \\
& c_{2}=\sqrt{R_{n n}} .
\end{aligned}
$$

It is important to note that the hyperbolicity of the system of equations is strongly tied to the requirement of weak realizability, a fact that has already been noticed with respect to incompressible fluids $[22,24]$. As to what concerns the convective subset (27) alone, weak realizability is automatically satisfied for regular $\left(C^{1}\right)$ solutions. Moreover, the Reynolds stress stays over-realizable if the initial state is over-realizable (see appendix A) so that eigenvalues $\lambda_{2}$ and $\lambda_{7}$ do not coincide with $\lambda_{3-6}$ (this property ensures the existence of a diagonal form of matrix $\mathbf{A}(\mathbf{W})$ ).

The set of eigenvalues differs visibly from the familiar case of gas dynamics. Waves associated with $\lambda_{1}$ and $\lambda_{8}$ are of acoustic nature, where $c_{1}$ signifies the celerity of isentropic density or pressure waves. This speed, however, depends on the (directional) turbulence intensity $R_{n n}$ in the present case.

The distinct speed of the 2- and 7-wave is a particular feature of the second-moment closure, $c_{2}$ playing a purely cinematic role. We are confronted with a system that permits the definition of two Mach numbers:

$$
\begin{aligned}
& \mathrm{M}_{1}=\frac{U_{n}}{c_{1}}=\frac{\mathrm{M}_{\text {Euler }}}{\sqrt{3 \mathrm{M}_{t}+1}}, \\
& \mathrm{M}_{2}=\frac{U_{n}}{c_{2}}=\frac{\mathrm{M}_{\text {Euler }}}{\mathrm{M}_{t}}
\end{aligned}
$$

where $\mathrm{M}_{\text {Euler }}=U_{n} / \sqrt{\gamma p / \rho}$ is the classical speed of sound and $\mathrm{M}_{t}=\sqrt{R_{n n}} / \sqrt{\gamma p / \rho}$ is a (directional) turbulent Mach number. The flow is supersonic (i.e. all waves have same sign) when $\left|\mathrm{M}_{1}\right|>1$. 

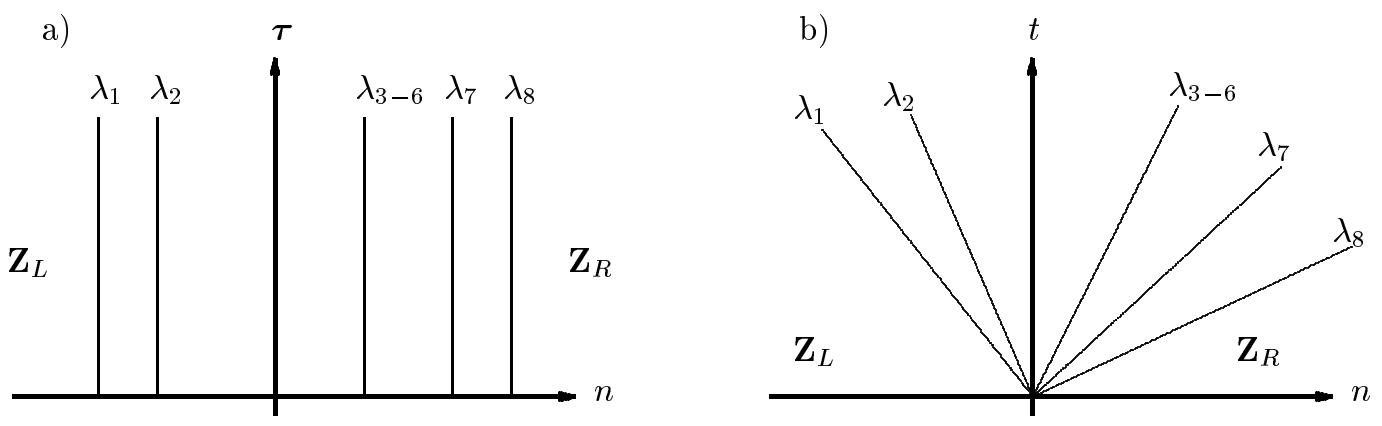

Figure 1: a) Quasi one-dimensional flow in the two dimensional plane $(\mathbf{n}, \boldsymbol{\tau})$ at an instant $t>0$. b) Wave propagation in space and time $(\mathbf{n}, t)$.

\subsection{The Riemann problem}

In the following we wish to analyze the one-dimensional Riemann problem which describes the flow developping from two semi-infinite states that have been separated initially by a discontinuity. Since we allow all components of the two-dimensional state vector to be non-zero a priori (but uniform in the tangential direction), the flow should really be termed "quasi one-dimensional". It is useful to work in local coordinates $(\mathbf{n}, \boldsymbol{\tau})$ perpendicular and tangential respectively to the discontinuity (see figure 1) so that we deal with the set of transformed variables

$$
\mathbf{Z}=\left(\rho, \rho U_{n}, \rho U_{t}, \rho E, \rho R_{n n}, \rho R_{t t}, \rho R_{s s}, \rho R_{n t}\right)^{t}
$$

where

$$
\begin{aligned}
& U_{n}=\mathbf{U}^{t} \mathbf{n}, \quad R_{n n}=\mathbf{n}^{t} \mathbf{R} \mathbf{n}, \quad R_{n t}=\mathbf{n}^{t} \mathbf{R} \boldsymbol{\tau} \\
& U_{t}=\mathbf{U}^{t} \boldsymbol{\tau}, \quad R_{t t}=\tau^{t} \mathbf{R} \boldsymbol{\tau}, R_{s s}=R_{33},
\end{aligned}
$$

The set of equations (27) can now be rewritten as follows:

$$
\mathbf{Z}_{, t}+\mathbf{A}_{n} \cdot \mathbf{Z}_{, n}=0, \quad \mathbf{A}_{n}=\frac{\partial \mathbf{F}_{n}}{\partial \mathbf{Z}}+\mathbf{C}_{n}^{n c}(\mathbf{Z})
$$

with initial data

$$
\mathbf{Z}(\mathbf{n}<0, t=0)=\mathbf{Z}_{L}, \quad \mathbf{Z}(\mathbf{n}>0, t=0)=\mathbf{Z}_{R} .
$$

\subsubsection{Genuinely non-linear and linearly degenerate waves}

In order to test for linearity of the different characteristic fields, the differential of the respective wave speed $\lambda_{i}$ needs to be projected upon the corresponding right eigenvector $\mathbf{r}^{i}$ [34]:

$$
\frac{\partial \lambda_{i}}{\partial \mathbf{Z}} \cdot \mathbf{r}^{i} \quad\left\{\begin{array}{lll}
=0 & \Rightarrow & \text { linearly degenerate field } i \\
\neq 0 & \Rightarrow & \text { genuinely non-linear field } i .
\end{array}\right.
$$

Eigenvectors $\mathbf{r}^{i}$ of our present system of equations are given in appendix B. It is straightforward to verify that the 1 - and 8-wave are genuinely non-linear (GNL), others are linearly degenerate (LD). 


\subsubsection{Riemann invariants}

Riemann invariants $I_{R}^{i}$ associated with the $i$ th field (satisfying $\left.\left(\partial I_{R}^{i} / \partial \mathbf{Z}\right) \cdot \mathbf{r}^{i}=0\right)$ are the following:

$$
\begin{aligned}
& I_{R}^{1}=\left\{p \rho^{-\gamma}, R_{n n} \rho^{-2}, U_{n}+\int \frac{c_{1}}{\rho} \mathrm{d} \rho,\left(R_{n n} R_{t t}-R_{n t}^{2}\right) / \rho^{2}, U_{t}+\int \frac{2 c_{1} R_{n t}}{c_{1}^{2}-R_{n n}} \frac{\mathrm{d} \rho}{\rho}, R_{s s},\right. \\
& \left.R_{s s}, R_{n t} \cdot \exp \left(-\frac{1}{3} \int \frac{c_{1}^{2}+R_{n n}}{c_{1}^{2}-R_{n n}} \frac{\mathrm{d} \rho}{\rho}\right)\right\} \\
& I_{R}^{2}=\left\{\rho, U_{n}, p, R_{n n}, U_{t}+\frac{R_{n t}}{\sqrt{R_{n n}}}, R_{n n} R_{t t}-R_{n t}^{2}, R_{s s}\right\} \\
& I_{R}^{3-6}=\left\{U_{n}, p+\rho R_{n n}, U_{t}, \rho R_{n t}\right\} \\
& I_{R}^{7}=\left\{\rho, U_{n}, p, R_{n n}, U_{t}-\frac{R_{n t}}{\sqrt{R_{n n}}}, R_{n n} R_{t t}-R_{n t}^{2}, R_{s s}\right\} \\
& I_{R}^{8}=\left\{p \rho^{-\gamma}, R_{n n} \rho^{-2}, U_{n}-\int \frac{c_{1}}{\rho} \mathrm{d} \rho,\left(R_{n n} R_{t t}-R_{n t}^{2}\right) / \rho^{2}, U_{t}-\int \frac{2 c_{1} R_{n t}}{c_{1}^{2}-R_{n n}} \frac{\mathrm{d} \rho}{\rho}, R_{s s},\right. \\
& \left.R_{n t} \cdot \exp \left(-\frac{1}{3} \int \frac{c_{1}^{2}+R_{n n}}{c_{1}^{2}-R_{n n}} \frac{\mathrm{d} \rho}{\rho}\right)\right\} \text {. }
\end{aligned}
$$

\subsubsection{Approximate jump conditions}

While deriving the exact jump conditions across discontinuities of familiar hyperbolic systems of conservation laws, e.g. in gas dynamics (Rankine-Hugoniot relations), extensive use of the conservation principle is made. It is not possible to proceed in an analogous way in the present case of a non-conservative set of equations. Instead, we resort to results from the theory of distributions. Le Floch [36] and Le Floch and Liu [37] have constructed a generalized Rankine-Hugoniot relation that depends on the path in state-space taken by the variables of the problem as a discontinuity is crossed. This approach is strictly valid only in the limit of weak shocks. Herein we suppose a linear path for variables $\mathbf{V}=\left(\rho^{-1}, U_{n}, U_{t}, p, \rho R_{n n}, \rho R_{t t}, \rho R_{s s}, \rho R_{n t}\right)^{t}$ (see also references [36, 13]), which leads to the following set of approximated jump conditions (appendix $\mathrm{C}$ for details):

$$
\begin{aligned}
& -\sigma[\rho]+\left[\rho U_{n}\right] \quad=0 \\
& -\sigma\left[\rho U_{n}\right]+\left[\rho U_{n}^{2}+\rho R_{n n}+p\right] \quad=0 \\
& -\sigma\left[\rho U_{t}\right]+\left[\rho U_{n} U_{t}+\rho R_{n t}\right]=0 \\
& -\sigma[\rho E]+\left[U_{n}\left(\rho E+\rho R_{n n}+p\right)+U_{t} \rho R_{n t}\right]=0 \\
& -\sigma\left[\rho R_{n n}\right]+\left[U_{n} \rho R_{n n}\right] \quad=-2 \overline{\left(\rho R_{n n}\right)}\left[U_{n}\right] \\
& -\sigma\left[\rho R_{t t}\right]+\left[U_{n} \rho R_{t t}\right] \quad=-2 \overline{\left(\rho R_{n t}\right)}\left[U_{t}\right] \\
& -\sigma\left[\rho R_{s s}\right]+\left[U_{n} \rho R_{s s}\right] \quad=0 \\
& -\sigma\left[\rho R_{n t}\right]+\left[U_{n} \rho R_{n t}\right] \quad=-\overline{\left(\rho R_{n n}\right)}\left[U_{t}\right]-\overline{\left(\rho R_{n t}\right)}\left[U_{n}\right] .
\end{aligned}
$$

We use standard notation for jumps $[\phi]=\phi_{r}-\phi_{l}$ and arithmetic means $\bar{\phi}=\left(\phi_{r}+\phi_{l}\right) / 2$ of a variable $\phi$ where $\phi_{r}$ and $\phi_{l}$ are states to the right and to the left of the respective discontinuity.

When dealing with LD fields the solution of the above jump conditions is equivalent to the corresponding Riemann invariants, i.e. $\left[I_{R}^{L D}\right]=0$, as is the case for conservation laws (cf. $[34,36]$ ). Furthermore, our approximate jump conditions are consistent with the classical Rankine-Hugoniot relations in the non-turbulent limit, which can easily be verified by setting all components of $\mathbf{R}$ to zero in (40). As to what concerns the GNL fields, shocks are described by the following parametrization $(\beta=(\gamma+1) /(\gamma-1))$ :

$$
\begin{aligned}
& \frac{\rho_{r}}{\rho_{l}}=z, \\
& \frac{p_{r}}{p_{l}}=\frac{\beta z-1}{\beta-z},
\end{aligned}
$$




$$
\begin{aligned}
\frac{\left(R_{n n}\right)_{r}}{\left(R_{n n}\right)_{l}} & =\frac{2 z-1}{z(2-z)}, \\
{\left[U_{n}\right] } & = \pm \frac{(z-1)}{\sqrt{z}} \sqrt{\frac{2 \gamma p_{l}}{\rho_{l}(\gamma-1)(\beta-z)}+\frac{3\left(R_{n n}\right)_{l}}{2-z}}
\end{aligned}
$$

and

$$
\sigma=\frac{z\left(U_{n}\right)_{r}-\left(U_{n}\right)_{l}}{z-1} .
$$

The sign of the velocity jump in equation (44) can be selected using the inviscid entropy inequality of section 2.3. By introducing (45) and (42) into relation (19) it is a simple matter to show that $\left[U_{n}\right] \leq 0$. Thus, in equation (44) the negative sign corresponds to a 1-shock $(z>1)$ and the positive sign to a 8 -shock $(z<1)$. The remaining solutions for jumps of variables $R_{t t}, R_{s s}, R_{n t}$ and $U_{t}$ are given in appendix $\mathrm{D}$.

\subsubsection{Approximate analytical solution}

Using the above results, the quasi one-dimensional Riemann problem may be solved by connecting left and right states $\mathbf{Z}_{L}$ and $\mathbf{Z}_{R}$ across the five distinct characteristic waves (cf. [34] for the basic method). We obtain the following solution:

Proposition 3: The one-dimensional Riemann problem associated with the nonconservative system (36), approximate jump conditions (40) and initial data (37) has a unique, realizable solution provided that the following condition holds

$$
\left(U_{n}\right)_{R}-\left(U_{n}\right)_{L}<X_{L}+X_{R},
$$

where

$$
X_{i}=\left(\frac{\gamma p_{i}}{\rho_{i}}\right)^{1 / 2} \int_{0}^{\rho_{i}}\left(\frac{a}{\rho_{i}}\right)^{\frac{\gamma-1}{2}}\left(1+3 \frac{\rho_{i}\left(R_{n n}\right)_{i}}{\gamma p_{i}}\left(\frac{a}{\rho_{i}}\right)^{3-\gamma}\right)^{1 / 2} \frac{\mathrm{d} a}{a},
$$

and provided that initial states $\mathbf{Z}_{L}$ and $\mathbf{Z}_{R}$ comply with weak realizability constraints (5), (7) and (8).

A proof for proposition 3 has been established in reference [38].

We remark that the upper limit for the initial velocity difference (46) is quite similar to the one encountered in the case of a first order closure ( $k-\varepsilon$ model, cf. [13]) and that this limit reduces to the expression known from gas dynamics (cf. [34]) if turbulence is set to zero. The integration in (47) can be carried out for the particular case of $\gamma=3$ where $X_{i}(\gamma=3)=2 /(\gamma-1)\left(c_{1}\right)_{i}$. As can be seen from the solution of the jump conditions, the strength of a shock wave is limited when using the hypothesis of a linear path. The admissible maximum, beyond which normal stresses become negative (equation (43)), is

$$
\frac{\max \left(\rho_{l}, \rho_{r}\right)}{\min \left(\rho_{l}, \rho_{r}\right)}=\min (\beta, 2) .
$$

We emphasize once again that even for subcritical shock strengths, the analytical solution is an approximation owing to the jump conditions. For regular solutions, however, the above results (Riemann invariants) are exact; moreover, the given relations across LD fields are valid in any case.

Finally, we point out that the positivity of density and pressure is preserved throughout the solution and that the Reynolds stress tensor remains realizable at all times (cf. [38]). 


\section{An approximate numerical Riemann solver}

\subsection{Introduction}

Most numerical solution strategies for flow problems of mixed hyperbolic-elliptic type are centered around the treatment of the hyperbolic part of the governing equations. It is our purpose in this section to present one such method for dealing with second-moment closures of the type defined in section 2.1. With the above presented analytical solution of the Riemann problem, it would in principle be possible and straightforward to construct a Godunov-type scheme as has been done successfully in the case of the $k-\varepsilon$ model [12]. However, the computational overhead produced by the solution of the non-linear problem (36) does in general not pay off in terms of physical realism. We will thus content ourselves with an approximate Riemann solver that is built upon the analytic solution to a linearized form of equation (36) similar to Roe's method [5]. Before presenting the flux formulation and integration method for the non-conservative system we quickly recall the basic method that has been created for conservation laws.

\subsection{Roe's scheme for systems of conservation laws}

Let us consider a hyperbolic system of conservation laws, viz

$$
\mathbf{Z}_{, t}+\mathbf{A}_{n}^{\text {cons }}(\mathbf{Z}) \cdot \mathbf{Z}_{, n}=0, \quad \mathbf{A}^{\text {cons }}=\frac{\partial \mathbf{F}}{\partial \mathbf{Z}}
$$

An approximate Riemann solver is such that the exact solution to the linearized problem

$$
\mathbf{Z}_{, t}+\mathcal{A}\left(\mathbf{Z}_{L}, \mathbf{Z}_{R}\right) \cdot \mathbf{Z}_{, n}=0
$$

is calculated which consists of five simple waves since all fields are LD. The corresponding numerical flux function is thus expressed by the well-known formula

$$
\mathcal{F}^{\text {Roe }}=\frac{1}{2}\left(\mathbf{F}_{L}+\mathbf{F}_{R}\right)-\frac{1}{2}\left|\mathcal{A}\left(\mathbf{Z}_{L}, \mathbf{Z}_{R}\right)\right| \cdot\left(\mathbf{Z}_{R}-\mathbf{Z}_{L}\right)
$$

The problem of finding a sensible linearization $\mathcal{A}\left(\mathbf{Z}_{L}, \mathbf{Z}_{R}\right)$ has been translated into three conditions of consistency by Roe [5]:

(i) $\mathcal{A}\left(\mathbf{Z}_{L}, \mathbf{Z}_{R}\right)$ is hyperbolic and a diagonal form exists,

(ii) $\mathcal{A}(\mathbf{Z}, \mathbf{Z})=\mathbf{A}^{\text {cons }}(\mathbf{Z})$,

(iii) $\mathcal{A}\left(\mathbf{Z}_{L}, \mathbf{Z}_{R}\right)[\mathbf{Z}]=[\mathbf{F}]$.

In the case of the Euler equations of gas dynamics, it turns out that the linearized system matrix is equal to the original system matrix under a transformation of variables that has been termed Roe's average:

$$
\mathcal{A}^{\text {Euler }}=\mathbf{A}^{\text {Euler }}\left(\mathbf{Z}^{\text {Roe }}\left(\mathbf{Z}_{L}, \mathbf{Z}_{R}\right)\right)
$$

In the past, some authors have forced the hyperbolic part of the second-moment closure equations to take a conservative form by eliminating from equation (20) the production term and the action of the Reynolds stress in the conservative flux of momentum and total energy [7, 11]. In the resulting truncated system turbulence is only felt via the pressure that is defined by equation (2). This simplified approach enables to simply use Roe's flux formulation (51) in conjunction with Roe's average for all variables (cf. [11] for details). For future reference, we term this procedure the 'decoupled approach'. 


\subsection{Roe-type scheme for non-conservative systems}

We focus once again on a non-conservative system of transport equations

$$
\mathbf{Z}_{, t}+\mathbf{A}_{n}(\mathbf{Z}) \cdot \mathbf{Z}_{, n}=0, \quad \mathbf{A}_{n}=\frac{\partial \mathbf{F}_{n}}{\partial \mathbf{Z}}+\mathbf{C}_{n}^{n c}(\mathbf{Z})
$$

As will be explained in the following (see section 4.4) we discretize the source term $\mathbf{C}_{n}^{n c}(\mathbf{Z}) \mathbf{Z}_{, n}$ in a simple, centered manner. As a consequence, applying Roe's flux-difference-splitting gives formally the same numerical flux formula as in the above case of conservation laws [11]:

$$
\mathcal{F}^{R N C}=\frac{1}{2}\left(\mathbf{F}_{L}+\mathbf{F}_{R}\right)-\frac{1}{2}\left|\mathcal{A}\left(\mathbf{Z}_{L}, \mathbf{Z}_{R}\right)\right| \cdot\left(\mathbf{Z}_{R}-\mathbf{Z}_{L}\right)
$$

Similarly, we require the linearization $\mathcal{A}$ to fulfil the following two fundamental constraints:

(i) $\mathcal{A}\left(\mathbf{Z}_{L}, \mathbf{Z}_{R}\right)$ is hyperbolic and a diagonal form exists,

(ii) $\mathcal{A}(\mathbf{Z}, \mathbf{Z})=\mathbf{A}_{n}(\mathbf{Z})$.

In a straightforward extension of Roe's above idea (iii), i.e. that the numerical flux be exact in the case of a shock wave being located between two nodes, one would write as the third condition

(iii) $)_{a} \mathcal{A}\left(\mathbf{Z}_{L}, \mathbf{Z}_{R}\right)[\mathbf{Z}]=[\mathbf{F}]+\overline{\mathbf{C}^{n c}}\left(\mathbf{Z}_{L}, \mathbf{Z}_{R}\right)[\mathbf{Z}]$

The jump conditions of the non-conservative source term $\overline{\mathbf{C}^{n c}}[\mathbf{Z}]$ are the approximate ones based on the assumption of a linear path (i.e. equation (40) for the present system).

In the case of $k-\varepsilon$ type closures [9, 39] and in the context of two-phase flows [6], conditions (i), (ii) and (iii) $)_{a}$ again lead to a linearized matrix resembling the original system matrix

$$
\mathcal{A}\left(\mathbf{Z}_{L}, \mathbf{Z}_{R}\right)=\mathbf{A}\left(\tilde{\mathbf{Z}}\left(\mathbf{Z}_{L}, \mathbf{Z}_{R}\right)\right)
$$

where $\tilde{\mathbf{Z}}$ is a particular average that differs from Roe's averaging.

In the present case, however, the linearized matrix $\mathcal{A}$ obtained from the above relations (i), (ii) and (iii) $)_{a}$ cannot be recast into the form of the system matrix $\mathbf{A}\left(\hat{\mathbf{Z}}\left(\mathbf{Z}_{L}, \mathbf{Z}_{R}\right)\right)$ since a corresponding average $\hat{\mathbf{Z}}$ does not exist [11]

$$
\nexists \quad \hat{\mathbf{Z}}\left(\mathbf{Z}_{L}, \mathbf{Z}_{R}\right) \quad / \quad \mathbf{A}(\hat{\mathbf{Z}})[\mathbf{Z}]=[\mathbf{F}]+\overline{\mathbf{C}^{n c}}\left(\mathbf{Z}_{L}, \mathbf{Z}_{R}\right)[\mathbf{Z}] .
$$

The matrix $\mathcal{A}$ that issues from condition (iii) ${ }_{a}$ is thus in a form not suitable for numerical purposes, in particular its diagonalization is very complicated. Consequently, we have relaxed this condition and replaced it by the following simple expression based on an arithmetic average:

(iii) $)_{b} \mathcal{A}\left(\mathbf{Z}_{L}, \mathbf{Z}_{R}\right)=\mathbf{A}(\mathbf{Z}(\overline{\mathbf{Y}}))$

where $\mathbf{Y}=\left(\rho, U_{n}, U_{t}, H_{t}, R_{n n}, R_{t t}, R_{s s}, R_{n t}\right)^{t}$ and total enthalpy being defined as $H_{t}=E+p / \rho$. Our numerical flux function can finally be written as ("RNC" designating "Roe Non Conservative"):

$$
\mathcal{F}^{R N C}=\frac{1}{2}\left(\mathbf{F}_{L}+\mathbf{F}_{R}\right)-\frac{1}{2}|\mathbf{A}(\mathbf{Z}(\overline{\mathbf{Y}}))| \cdot\left(\mathbf{Z}_{R}-\mathbf{Z}_{L}\right)
$$

The "absolute value" of the system matrix is calculated through the following relation

$$
|\mathbf{A}(\mathbf{Z}(\overline{\mathbf{Y}}))|=\mathcal{R}(\mathbf{Z}(\overline{\mathbf{Y}})) \cdot|\Lambda(\mathbf{Z}(\overline{\mathbf{Y}}))| \cdot \mathcal{R}^{-1}(\mathbf{Z}(\overline{\mathbf{Y}}))
$$

where diagonalization matrices $\mathcal{R}$ and $\mathcal{R}^{-1}$ are given in appendix $\mathrm{B}$ and $\Lambda$ is the diagonal eigenvalue matrix. 


\subsection{Integration method}

Since in most finite volume methods multidimensional flows are treated as a succession of quasi one-dimensional problems for each cell face, it suffices in the following to present a one-dimensional discretization, keeping the notation of section 3.3.

Integrating equation (53) over a finite volume $\Omega_{i}\left(V_{\Omega_{i}}\right.$ being the cell volume, $\Gamma_{i}$ designating the cell surface, $\Delta t$ the time step, superscript $n$ indexing time steps) we obtain:

$$
V_{\Omega_{i}} \cdot\left(\mathbf{Z}_{i}^{n+1}-\mathbf{Z}_{i}^{n}\right)+\Delta t\left\{\oint_{\Gamma_{i}} \mathcal{F}^{R N C}\left(\mathbf{Z}^{n}\right) \mathrm{d} \Gamma_{i}+\mathbf{S}_{i}\left(\mathbf{Z}^{n}\right)\right\}=0 .
$$

The source terms $\mathbf{S}_{i}$ are expressed by a centered difference, so that

$$
\mathbf{S}_{i}=\int_{\Omega_{i}} \mathbf{C}^{n c}(\mathbf{Z}) \cdot \mathbf{Z}_{, n} \mathrm{~d} \Omega=\mathbf{C}^{n c}\left(\mathbf{Z}_{i}^{n}\right) \cdot \int_{\Omega_{i}} \mathbf{Z}_{, n} \mathrm{~d} \Omega=\mathbf{C}^{n c}\left(\mathbf{Z}_{i}^{n}\right) \cdot \oint_{\Gamma_{i}} \mathcal{Z}_{\Gamma}\left(\mathbf{Z}^{n}\right) \mathrm{d} \Gamma_{i}
$$

where

$$
\mathcal{Z}_{L R}\left(\mathbf{Z}^{n}\right)=\frac{\mathbf{Z}_{i}^{n}+\mathbf{Z}_{j}^{n}}{2}
$$

with $\mathbf{Z}_{j}$ being the local neighbouring node at the respective partial cell face $\Gamma_{i j} \in \Gamma_{i}$.

\section{Some numerical experiments}

\subsection{Generalities}

In the following we present some numerical results of quasi one-dimensional flow problems that demonstrate important properties of the proposed method. We consider two Riemann problems with different initial states as detailled in table I . These give rise to a turbulent shock-tube flow and a symmetrical double shock configuration respectively. At this point, two features of the chosen initial values should be noted. Firstly, turbulence intensity is high enough for the particularities of the system of equations to be felt (with respect to the Euler equations). Secondly, the Reynolds stresses $\mathbf{R}_{L}$ and $\mathbf{R}_{R}$ are in an anisotropic state since otherwise the "new" pair of waves $\lambda_{2}$ and $\lambda_{7}$ would be "invisible" as a consequence of the form of the respective invariants (cf. (39)).

The length of the domain $L$, the position of the diaphragm $x_{0}$ and total integration time $t_{f i n}$ are given in table II . A number of 500 grid nodes are equally spaced over the distance $L$ in all examples shown below. This spatial resolution suffices for the present investigation of numerical wave propagation (in an engineering application a higher order extension of the scheme, e.g. references [40,41], should be used). The time step used for the computations obeys the condition of non-interaction of waves originating from neighboring cell faces [4], thus

$$
\frac{\Delta t}{\Delta x} \cdot \max \left(\left|\lambda_{i}\right|\right) \leq 0.5
$$

All calculations are performed with the ratio of specific heats $\gamma$ taking a value of $7 / 5$.

\subsection{Turbulent shock-tube flow}

This case is identical to Sod's problem [42] as far as initial values of mean quantities are concerned. The flow does develop in a way that is similar to the laminar or gas dynamics counterpart with a rarefaction wave moving to the left and a shock wave propagating to the right followed by three distinct LD waves of which the wave in the center corresponds to the contact discontinuity. In 


\begin{tabular}{|c|c|c|c|c|c|c|c|c|c|c|c|}
\hline & $\rho\left[\frac{\mathrm{kg}}{\mathrm{m}^{3}}\right]$ & $U_{n}\left[\frac{\mathrm{m}}{\mathrm{s}}\right]$ & $U_{t}\left[\frac{\mathrm{m}}{\mathrm{s}}\right]$ & $p\left[\frac{\mathrm{kg}}{\mathrm{m} \mathrm{s}^{2}}\right]$ & $R_{n n}\left[\frac{\mathrm{m}^{2}}{\mathrm{~s}^{2}}\right]$ & $R_{t t}\left[\frac{\mathrm{m}^{2}}{\mathrm{~s}^{2}}\right]$ & $R_{n t}\left[\frac{\mathrm{m}^{2}}{\mathrm{~s}^{2}}\right]$ & $R_{s s}\left[\frac{\mathrm{m}^{2}}{\mathrm{~s}^{2}}\right]$ & $b_{n t}$ & $\mathrm{M}_{t}$ \\
\hline \hline \multicolumn{10}{|c|}{ case 1 - turbulent shock-tube flow } \\
\hline $\mathrm{L}$ & 1 & 0 & 0 & $10^{5}$ & $\frac{2}{3} 10^{5}$ & $\frac{2}{3} 10^{5}$ & $\frac{1}{4} 10^{5}$ & $\frac{2}{3} 10^{5}$ & $\frac{1}{8}$ & 0.69 \\
\hline $\mathrm{R}$ & $\frac{1}{8}$ & 0 & 0 & $10^{4}$ & $\frac{16}{3} 10^{3}$ & $\frac{16}{3} 10^{3}$ & $2 \cdot 10^{3}$ & $\frac{16}{3} \cdot 10^{3}$ & $\frac{1}{8}$ & 0.22 \\
\hline \multicolumn{10}{|c|}{ case 2 double shock configuration } \\
\hline $\mathrm{L}$ & 1 & 100 & 0 & $10^{5}$ & $\frac{2}{3} 10^{4}$ & $\frac{2}{3} 10^{4}$ & $\frac{1}{4} 10^{4}$ & $\frac{2}{3} 10^{4}$ & $\frac{1}{8}$ & 0.22 \\
\hline $\mathrm{R}$ & 1 & -100 & 0 & $10^{5}$ & $\frac{2}{3} 10^{4}$ & $\frac{2}{3} 10^{4}$ & $\frac{1}{4} 10^{4}$ & $\frac{2}{3} 10^{4}$ & $\frac{1}{8}$ & 0.22 \\
\hline
\end{tabular}

Table 1: Initial conditions for quasi one-dimensional test problems. Tangential anisotropy is indicated by $b_{n t}=R_{n t} / R_{i i}$.

\begin{tabular}{|c|c|c|}
\hline & case 1 & case 2 \\
\hline \hline$L[m]$ & 30 & 30 \\
\hline$x_{0}[m]$ & 14 & 14 \\
\hline$t_{\text {fin }}\left[10^{-2} s\right]$ & 2.1 & 2.3 \\
\hline
\end{tabular}

Table 2: Some physical details concerning the simulation: length of the domain of integration $L$, position of the diaphragm $x_{0}$ and final integration time $t_{\text {fin }}$.

figure 2 all five waves are visible through the variation of tangential momentum $\rho U_{t}$. Density $\rho$ and normal velocity $U_{n}$ (see figures 3 and 4 ) behave qualitatively in the same manner as in Sod's original shock-tube flow with variations only across the 1-, 3-4-5-6- and 8-fields (1- and 8-fields respectively).

Figure 5 shows that the mean pressure changes considerably in the present case as the contact discontinuity is crossed due to non-negligible turbulence intensity. In accordance with theory (equations (39)) the sum of the normal stresses $p+\rho R_{n n}$ remains constant across all LD fields.

As a consequence of the coupling between Reynolds steresses and mean quantities, the tangential velocity $U_{t}$ takes on considerable values in the flow field (figure 6). On the same graph, we can clearly identify the location of the particular 2- and 7-waves (however smeared they might be) by comparing the behaviour of $U_{t}$ with the one of its corresponding invariants $U_{t} \pm R_{n t} / \sqrt{R_{n n}}$ that are also included.

All four Reynolds stress components are shown in figure 7 . The direct effect of production on $R_{n n}$ and $R_{n t}$ can be observed as well as the purely passive role of the spanwise normal stress $R_{s s}$ (justifying the designation as 'contact discontinuity' for the 3-4-5-6-wave).

Finally, figure 8 allows us to verify the correct numerical representation of other invariant expressions. We note the positivity of the determinant $\delta_{2}^{3}$ throughout the domain as well as the monotonous results for the two entropy-like ratios $p / \rho^{\gamma}$ and $R_{n n} / \rho^{2}$.

\subsection{Symmetrical double shock configuration}

In the second case, two shock waves, one left-running and one right-running, form due to initially impacting velocities $\left(U_{n}\right)_{L}$ and $\left(U_{n}\right)_{R}$ as can be seen from the distribution of normal velocity in figure 9. This configuration is of particular relevance to engineering problems in as far as it is an 
idealization of the transient state near a wall during the initial phase of a numerical simulation. The 3-4-5-6 contact discontinuity remains stationary in this case of symmetrical flow. Its position is marked by a localized loss of monotony of most variables in figures 11 to 14, e.g. density, pressure, Reynolds stress components. This feature of the numerical solution is not due to the current flux formulation (RNC) since it is also observed in similar symmetrical flow cases when using Godunov's method to solve the equations of gas dynamics or in conjunction with a $k$ - $\varepsilon$ type closure [12]. The small 'glitch' is due to the fact that an initially created perturbation at the point $x_{0}$ is not smoothed out since the eigenvalues $\lambda_{3-6}$ vanish there.

Once again invariants $U_{t} \pm R_{n t} / \sqrt{R_{n n}}$ (figure 14) indicate the position of the 2- and 7-waves which propagate in opposite directions.

Figures 15 to 18 show results obtained using the simple 'decoupled approach' (section 4.2) based on artificially rendering the hyperbolic subsystem conservative. Using this numerical solution method, dramatic oscillations occur for those variables that are subject to variation across waves 2 and 7, i.e. $U_{t}, R_{t t}$ and $R_{n t}$. Corresponding invariants are not preserved, as can be seen from figure 16. This non-physical bahaviour is a direct consequence of ignoring the particular form of the characteristics of the second-moment closure equations. The decoupled scheme is effectively "blind" with respect to the 2- and 7-wave. Hence, whenever these waves occur in the solution, no upwind contribution is introduced into the numerical flux by the scheme leading to instability (see appendix E for an explanation of this behaviour via von Neumann analysis).

\section{Conclusion}

We have investigated a class of simple second-moment closures for the turbulent stress tensor ensuring a realizable, objective system of modelled equations and allowing for a clear entropy inequality. The associated first order differential subset (containing convection and production mechanisms) cannot be cast into conservation form. Its hyperbolicity is assured for over-realizable initial fields. The system of characteristic waves is found to be quite distinct from the case of pure gas dynamics with a pair of additional LD waves appearing and a modification of the speed of sound due to turbulence.

Our analysis of the Riemann problem leads to a majority of exact partial solutions, i.e. across all LD waves and across regular GNL fields (Riemann invariants). These theoretical ingredients enable subsequent scrutinization of numerical results. In order to solve for variations across shock waves, we have resorted to approximate jump conditions based on a linear path in state-space. The resulting analytical solution of the complete Riemann problem is thus an approximation whose validity is limited to weak shocks.

We have proposed an approximate numerical Riemann solver of the flux-difference-splitting type to be used in finite volume simulation codes. For practical reasons it was not possible to enforce Roe's condition of consistency for stationary shock waves in our numerical flux function for the non-conservative system (RNC). Our results of quasi one-dimensional test calculations document the potential of the present method: solutions behave in the expected monotonous manner even in the presence of strong shocks, high turbulence intensity and anisotropy; theoretically deduced invariant expressions are numerically respected.

On the other hand, our calculations using an essentially Euler-based 'decoupled approach' lead to spurious oscillations at high turbulent Mach numbers. Simplifying the numerical task by neglecting to a large extent the influence of turbulence on the propagation properties of the second-order system should thus not be considered as an alternative. Use of the full, coupled, non-conservative subset is advised for accurate and stable time-dependent numerical simulations of the second-moment equations. 


\section{References}

[1] B.E. Launder. Turbulence modelling for the nineties: Second moment closure ... and beyond? In Twelfth Int. Conf. Num. Fluid Dynamics, 1990.

[2] C.G. Speziale. Analytical methods for the development of Reynolds stress closures in turbulence. Ann. Rev. Fluid Mech., 23:107-157, 1991.

[3] J.L. Lumley. Some comments on turbulence. Phys. Fluids A, 2:203-211, 1992.

[4] S.K. Godunov. A difference method for numerical calculation of discontinuous equations of hydrodynamics. Math. Sb., 47:217-300, 1959.

[5] P.L. Roe. Approximate Riemann solvers, parameter vectors, and difference schemes. J. Comp. Physics, 43, 1981.

[6] L. Combe and J.-M. Hérard. Un schéma volumes finis pour la simulation d'un modèle bifluide d'écoulements diphasiques compressibles gaz-solide. Revue Européenne des Eléments finis, 5(2):197-231, 1997.

[7] J.H. Morrison. A compressible Navier-Stokes solver with two-equation and Reynolds stress turbulence closure models. Report No. CR 4440, NASA, 1992.

[8] F. Ladeinde. Supersonic flux-split procedure for second moments of turbulence. AIAA J., $33(7): 1185-1195,1995$.

[9] A. Forestier, J.-M. Hérard, and X. Louis. Exact or approximate Riemann solvers to compute a two-equation turbulent compressible model. In Finite Elements in Fluids, New Trends and Applications, Venezia, Italy, 1995.

[10] A. Page and M. Uhlmann. Traitement de la partie hyperbolique du système des équations Navier-Stokes moyennées et des équations de transport issues d'une fermeture au premier ordre pour un fluide compressible. Technical Report 96-1, LMFA, Ecole Centrale de Lyon, France, Mars 1996. http://cephee.mecaflu.ec-lyon.fr/MFN/publi/publi.html.

[11] M. Uhlmann. Etude de modèles de fermeture au second ordre et contribution à la résolution numérique des écoulements turbulents compressibles. PhD Thesis, Ecole Centrale de Lyon, 1997.

[12] X. Louis. Modélisation numérique de la turbulence compressible. PhD Thesis, Université Paris VI, 1995.

[13] A. Forestier, J.-M. Hérard, and X. Louis. Solveur de type Godunov pour simuler les écoulements compressibles. C. R. Acad. Sci. Paris, t. 324, Série I:919-926, 1997.

[14] A. Favre. Equations des gaz turbulents, parties I et II. J. de Mécanique, 4(3):362-421, 1965.

[15] D. Vandromme. Turbulence modelling for compressible flows and implementation in NavierStokes solvers. Lecture series, von Karman Institute for Fluid Dynamics, 1991.

[16] S. Sarkar and B. Lakshmanan. Application of a Reynolds stress turbulence model to the compressible shear layer. AIAA J., 29:743-749, 1991.

[17] J.L. Lumley. Computational modelling of turbulent flows. Advances in Applied Mechanics, 18:123-176, 1978.

[18] B.E. Launder and D.B. Spalding. The numerical computation of turbulent flows. Comp. Meth. App. Mech. Eng., 3:269-289, 1974. 
[19] S.B. Pope. PDF methods for turbulent reactive flows. Prog. Energy Combust. Sci., 11:119192, 1985.

[20] T.-H. Shih and J.L. Lumley. Modelling of pressure correlation terms in Reynolds stress and scalar flux equations. Technical Report FDA-85-3, Cornell University, 1985.

[21] S. Fu, B.E. Launder, and D.P. Tselepidakis. Accommodating the effects of high strain rates in modelling the pressure-strain correlation. Technical Report TFD/87/5, UMIST Mechanical Engineering Dept., 1987.

[22] J.M. Hérard. Basic analysis of some second moment closures. Part I: incompressible isothermal turbulent flows. Theoret. Comput. Fluid Dynamics, 6(4):213-233, 1994.

[23] J.-M. Hérard. Suitable algorithms to preserve the realisability of Reynolds stress closures. In $A S M E F E D$ 215, 2nd joint ASME/JSME Fluids Engineering Division Summer Meeting, pages 73-80, Hilton Head, SC, USA, August 1995.

[24] J.-M. Hérard. Modèles au second ordre réalisables non dégénérés pour les écoulements turbulents incompressibles. C. R. Acad. Sci. Paris, t. 322, Série II b, 1996.

[25] T.-H. Shih, A. Shabbir, and J.L. Lumley. Realizability in second-moment turbulence closures revisited. Technical Report 106469, NASA, 1994.

[26] C.G. Speziale. Invariance of turbulent closure models. Phys. Fluids, 22(6):1033-1037, 1979.

[27] D. Vandromme. Contribution à la modélisation et á la prédiction d'écoulements turbulents à masse volumique variable. Thèse d'etat, Universite de Lille, 1983.

[28] M.W. Rubesin. Extra compressibility terms for Favre averaged two-equation models of inhomogeneous turbulent flows. Technical Report CR-177556, NASA, 1990.

[29] O. Zeman. On the decay of compressible isotropic turbulence. Phys. Fluids A, 3:951-955, 1991.

[30] S. Sarkar, G. Erlebacher, and M.Y. Hussaini. Compressible homogeneous shear: Simulation and modelling. Technical Report 92-6, ICASE, 1992.

[31] A.M. El-Baz and B.E. Launder. Second-moment modelling of compressible mixing layers. In W. Rodi and F. Martinelli, editors, Engineering Turbulence Modelling and Experiments 2, pages $63-72,1993$.

[32] O. Zeman. Compressible turbulence subjected to shear and rapid compression. In Durst et al., editor, Turbulent Shear Flows 8. Springer, 1993.

[33] J.R. Ristorcelli. A representation for the turbulent mass flux contribution to Reynolds stress and two-equation closures for compressible turbulence. Technical Report 93-88, NASA ICASE, 1993.

[34] J. Smoller. Shock waves and reaction diffusion equations. Springer, 1983.

[35] P.D. Lax. Hyperbolic systems of conservation laws and the mathematical theory of shock waves. CBMS Monographs. SIAM, Philadelphia, 1973.

[36] P. Le Floch. Entropy weak solutions to nonlinear hyperbolic systems under nonconservative form. Commun. In Partial Differential Equations, 13(6):669-727, 1988.

[37] P. Le Floch and T.-P. Liu. Existence theory for nonlinear hyperbolic systems in nonconservative form. Report No. 254, Ecole Polytechnique, Centre de Math. App., 1992. 
[38] C. Berthon, F. Coquel, J.-M. Hérard, and M. Uhlmann. An approximate solution of the Riemann problem for a realisable second-moment closure. submitted to Shock Waves, also as Report No. HE-41/98/054/A, Electricité de France.

[39] T. Buffard and J.-M. Hérard. Approximate Riemann solvers to compute turbulent compressible one and two-equation models. In ASME FED 238, ASME Fluids Engineering Division Summer Meeting, pages 189-196, San Diego, CA, USA, July 1996.

[40] B. van Leer. Towards the ultimate conservative difference scheme. V. A second-order sequel to Godunov's method. J. Comp. Phys., 32:101-136, 1979.

[41] H. Steve. Schemas Implicites Linéarises Décentrés pour la Résolution des Equations d'Euler en Plusieurs Dimensions. PhD Thesis, L'Universite de Provence Aix-Marseille 1, Juillet 1988.

[42] G.A. Sod. A survey of several finite difference methods for systems of nonlinear hyperbolic conservation laws. J. Comp. Phys., 27:1-31, 1978.

[43] C.G. Hirsch. Numerical computation of internal and external flows. J. Wiley, 1990. 


\section{Figures}

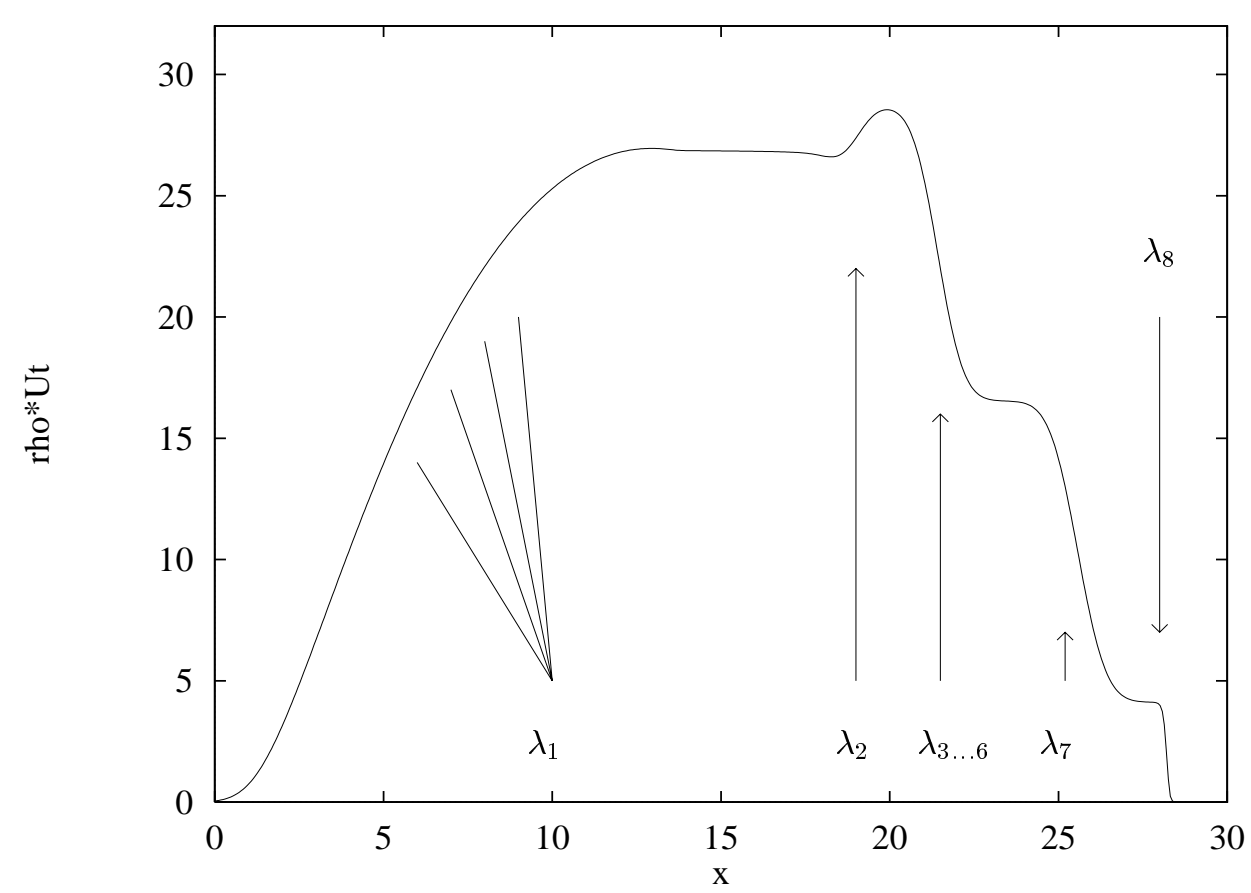

Figure 2: Turbulent shock tube problem, case 1; obtained distribution of tangential momentum $\rho U_{t}$ through which all five distinct waves of the problem become visible.

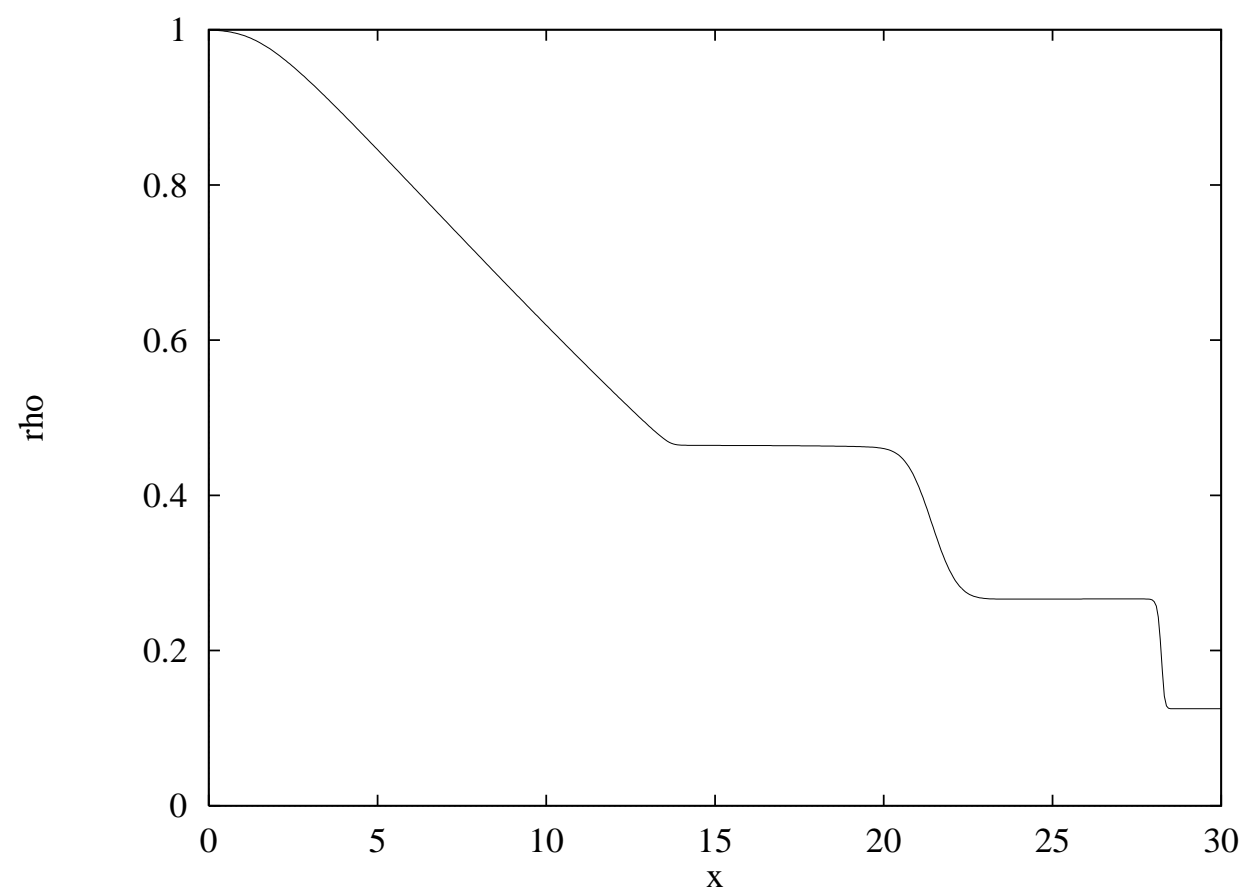

Figure 3: Case 1; distribution of density $\rho$ obtained with the present scheme (RNC). 


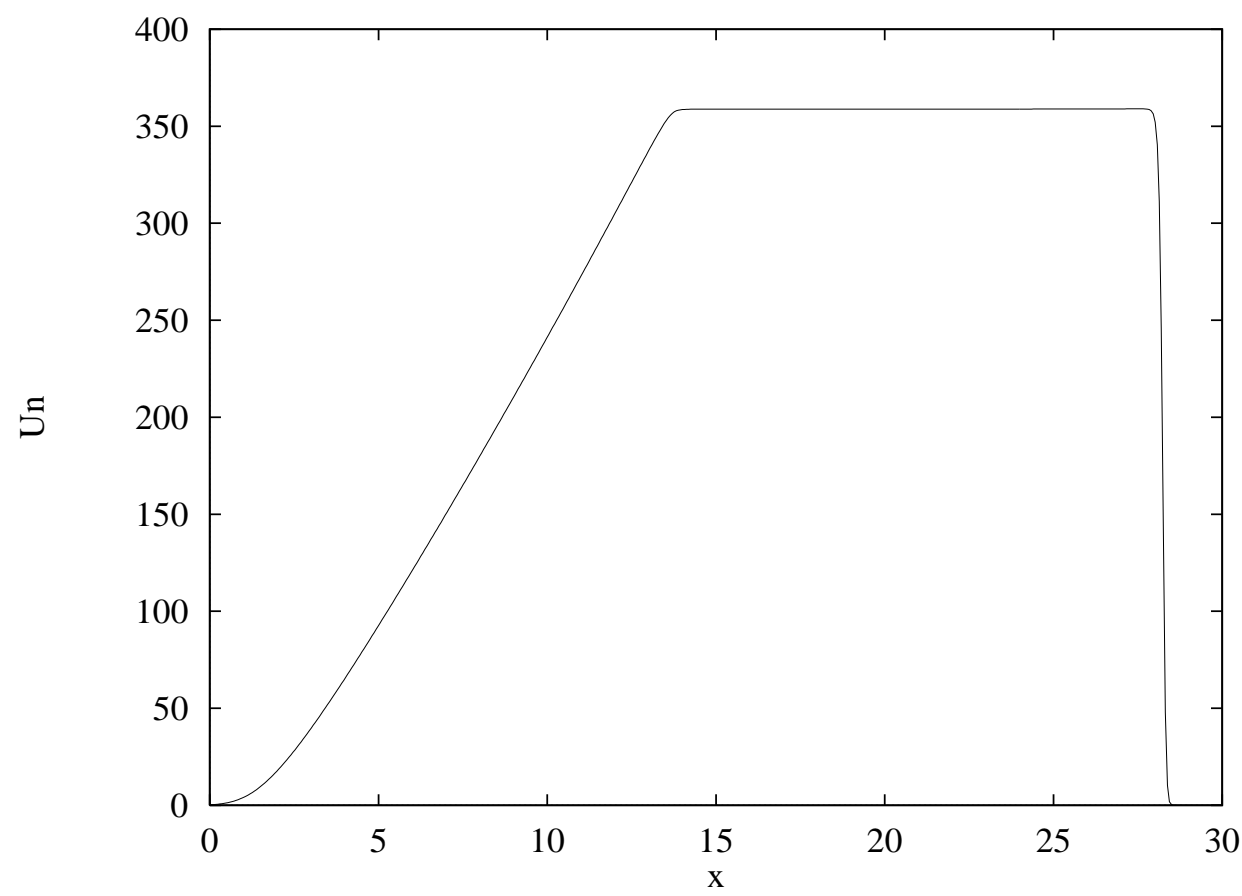

Figure 4: Case 1; distribution of normal velocity $U_{n}$ obtained with the present scheme (RNC).

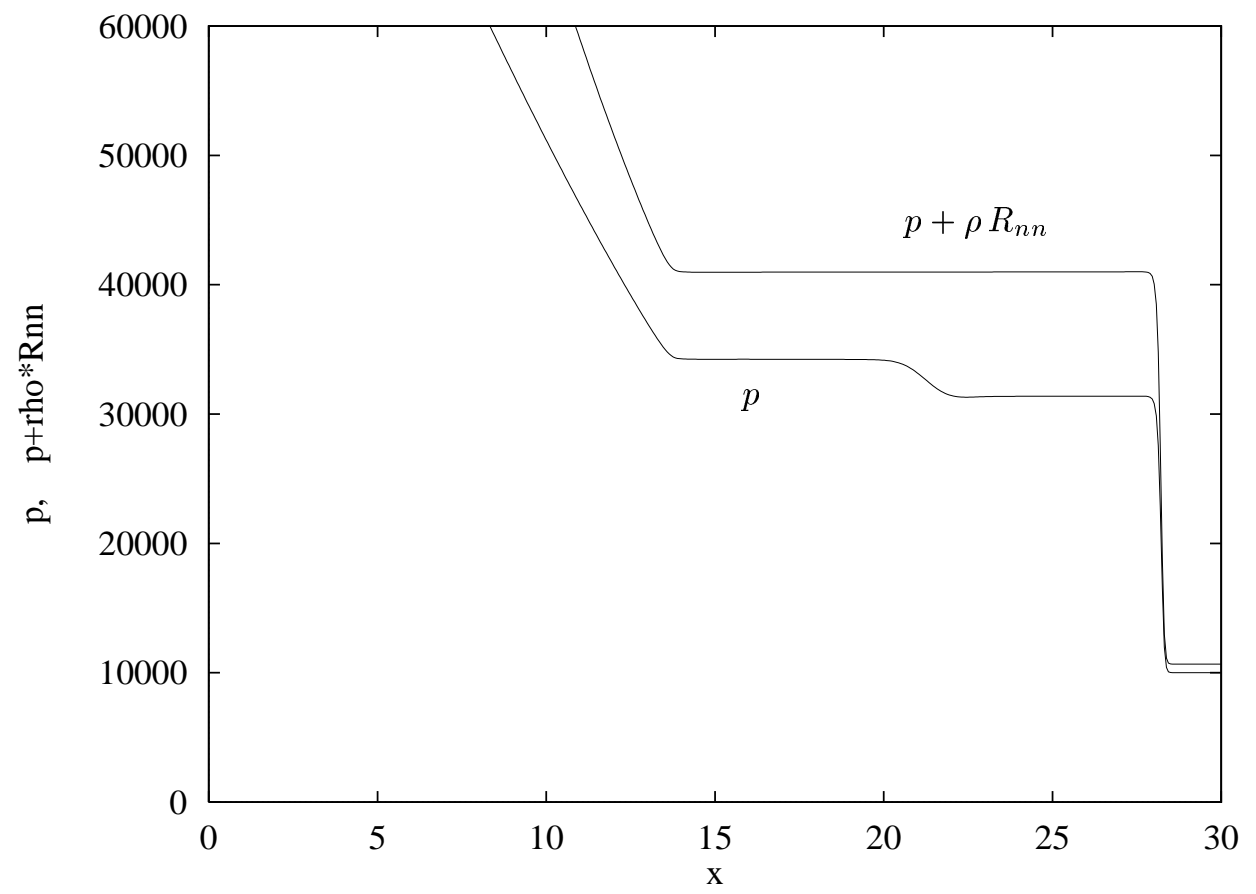

Figure 5: Case 1; distribution of pressure $p$ and total normal stress $p+\rho R_{n n}$, the latter being invariant with respect to the contact discontinuity in the center (3-4-5-6-wave). 


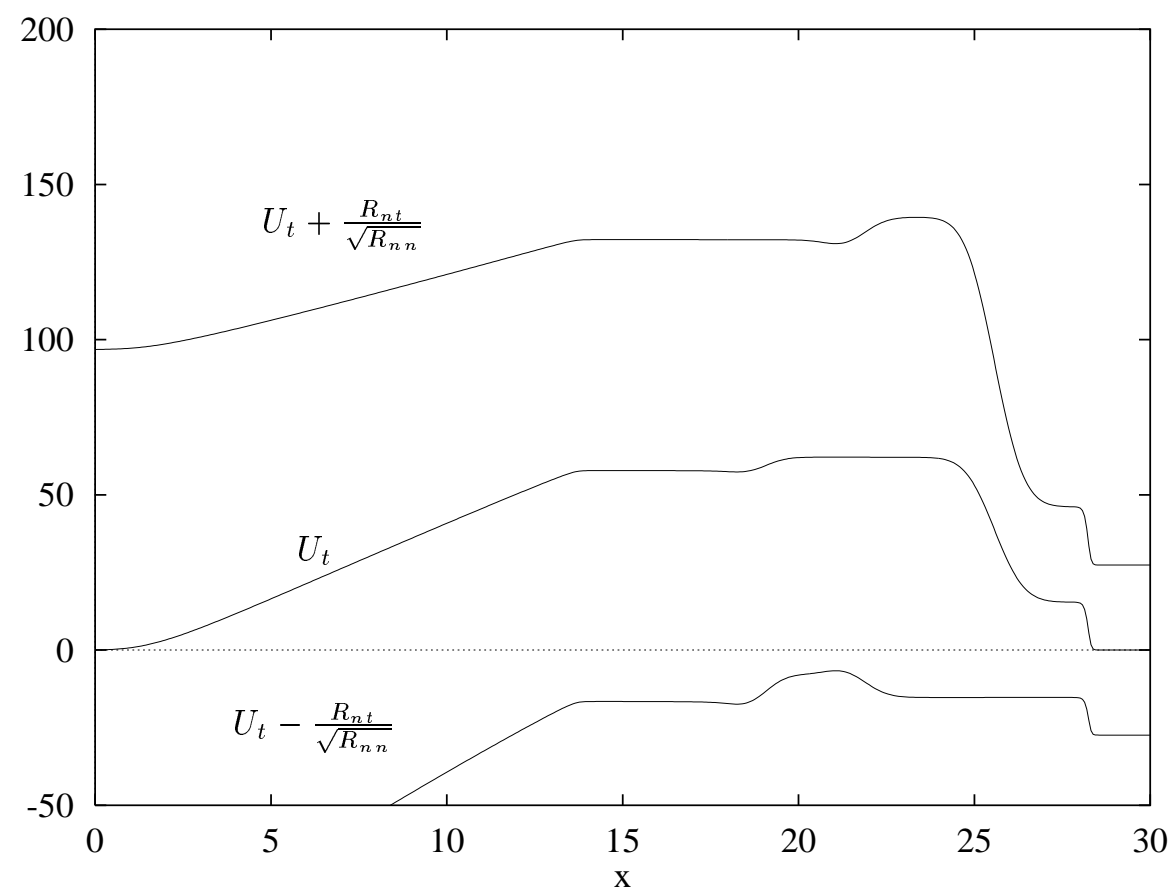

Figure 6: Case 1; distribution of tangential velocity $U_{t}$ and invariants $U_{t} \pm R_{n t} / \sqrt{R_{n n}}$ allowing to identify the location of the 2 - and 7 -wave. 
(a)

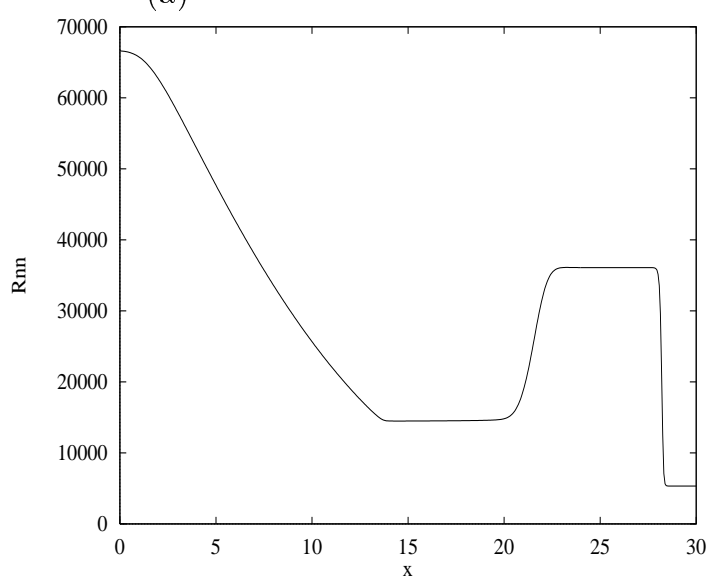

(c)

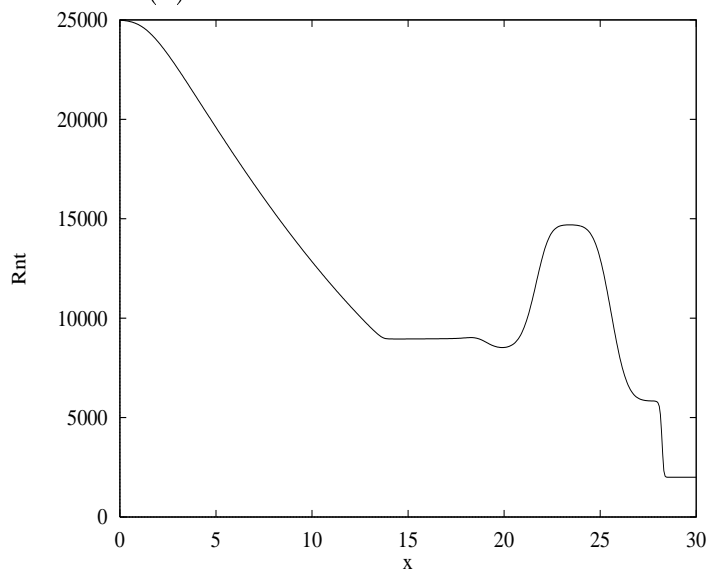

(b)

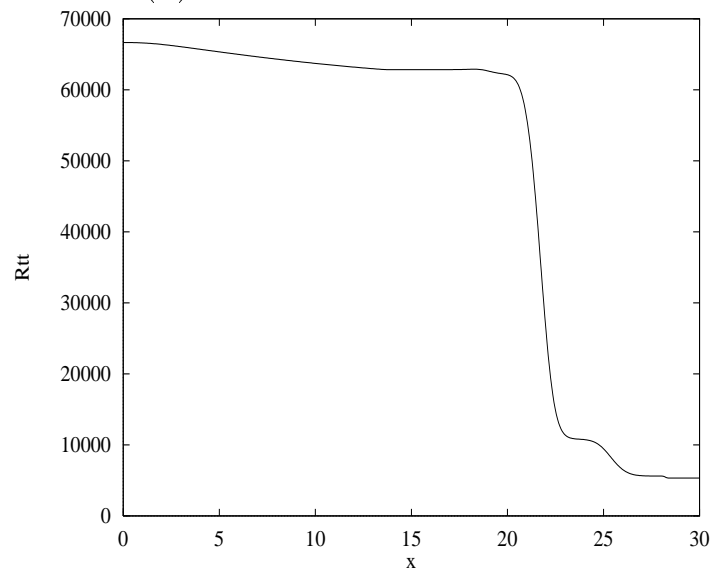

(d)

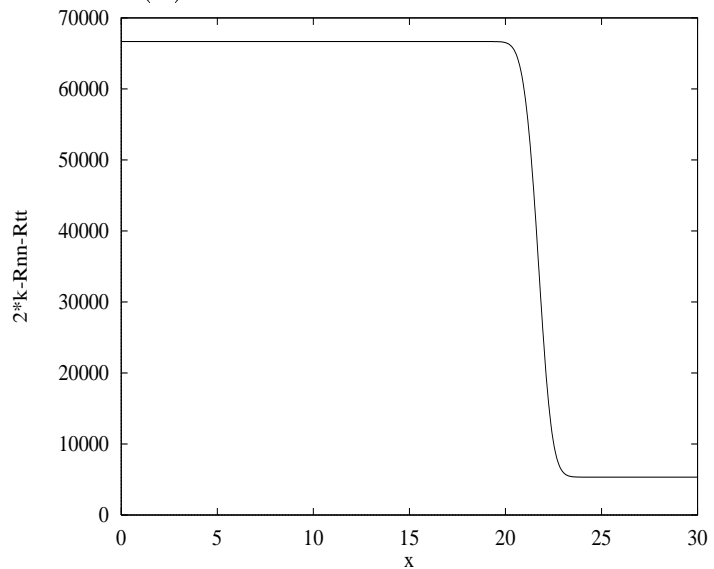

Figure 7: Case 1; distribution of Reynolds stress components obtained with the present scheme (RNC); (a) $R_{n n}$, (b) $R_{t t}$, (c) $R_{n t}$ and (d) $R_{s s}$. 
(a)

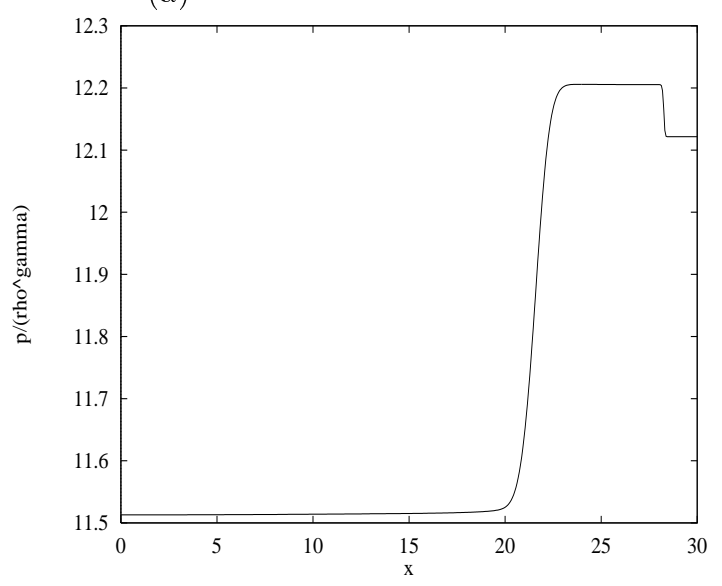

(c)

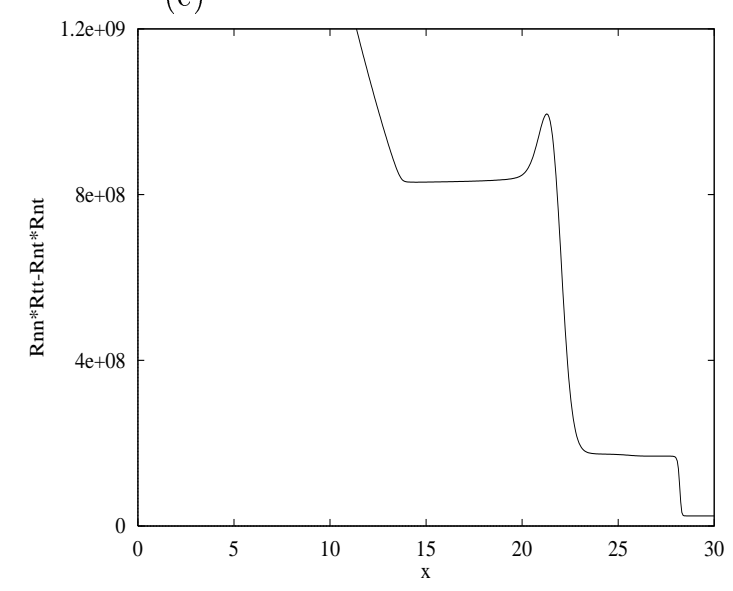

(b)

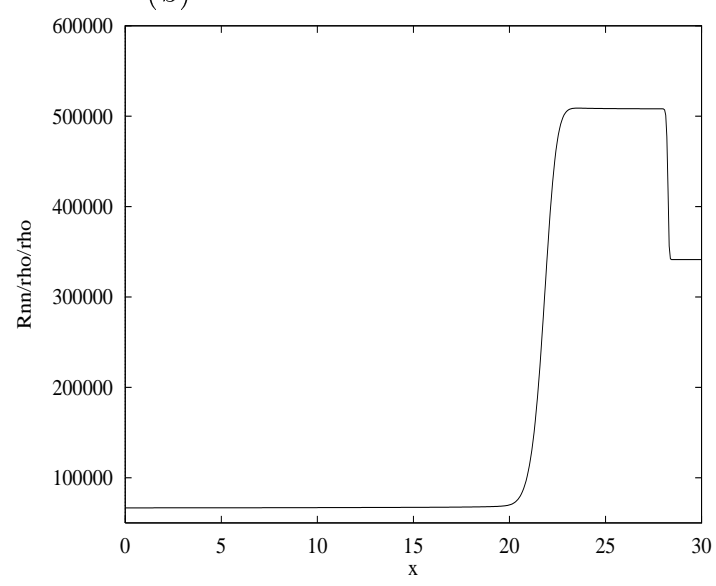

(d)

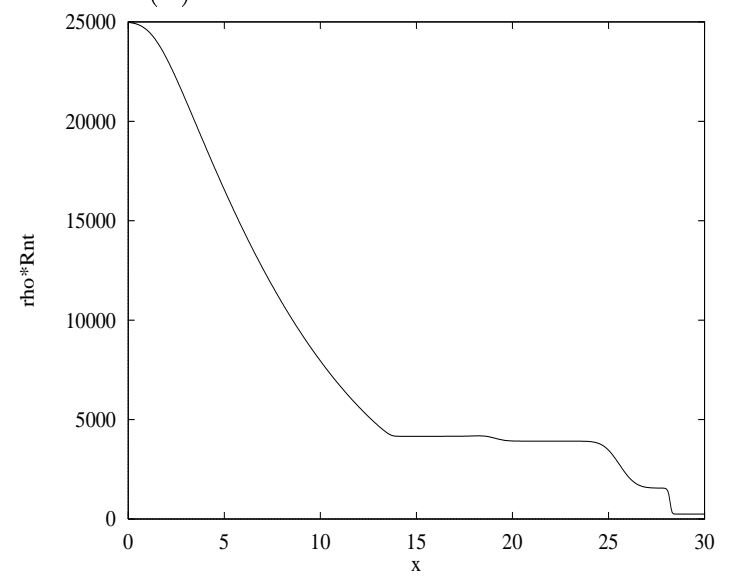

Figure 8: Case 1; distribution of different invariant expressions obtained with the present scheme (RNC); (a) $p / \rho^{\gamma}$, (b) $R_{n n} / \rho^{2}$, (c) $\delta_{2}^{3}$, (d) $\rho R_{n t}$. 


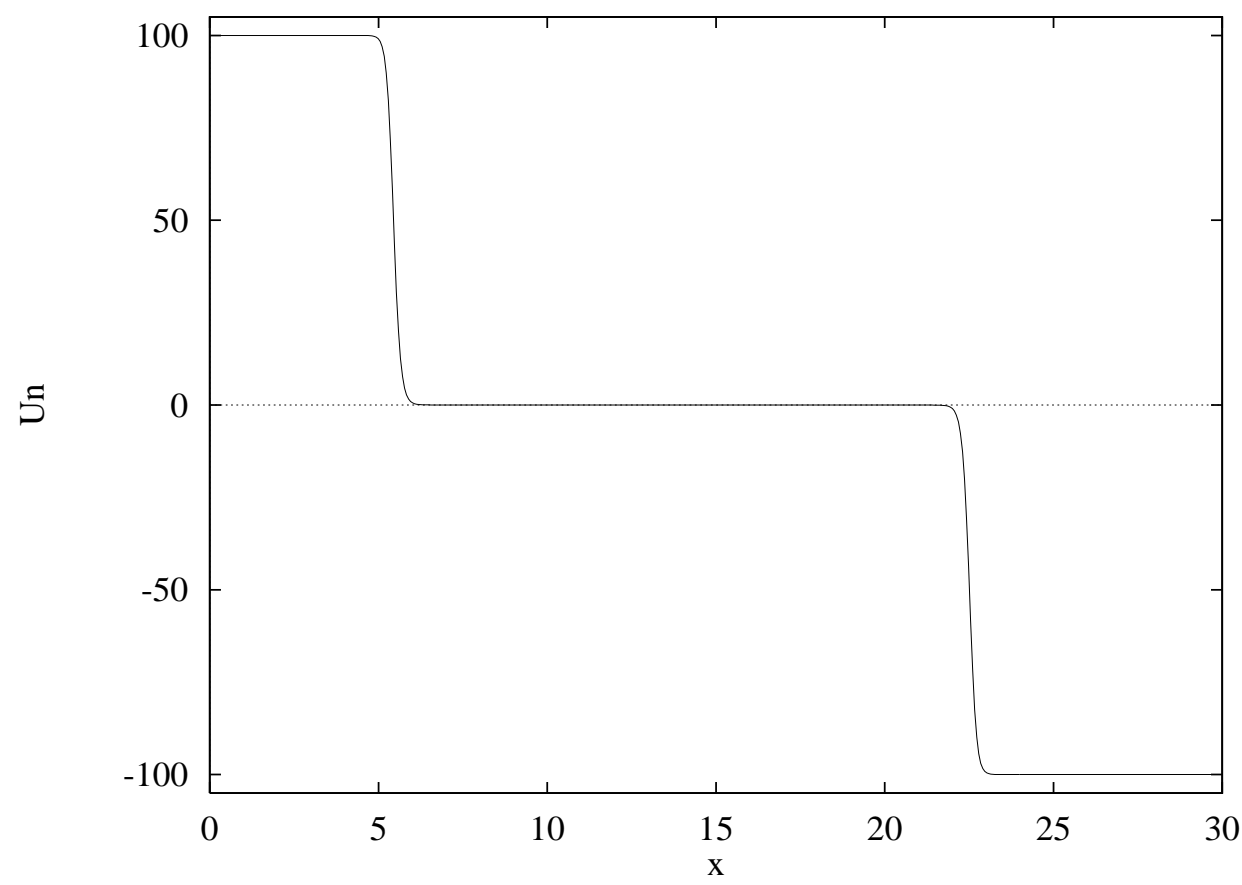

Figure 9: Case 2; distribution of normal velocity $U_{n}$ obtained with the present scheme (RNC).

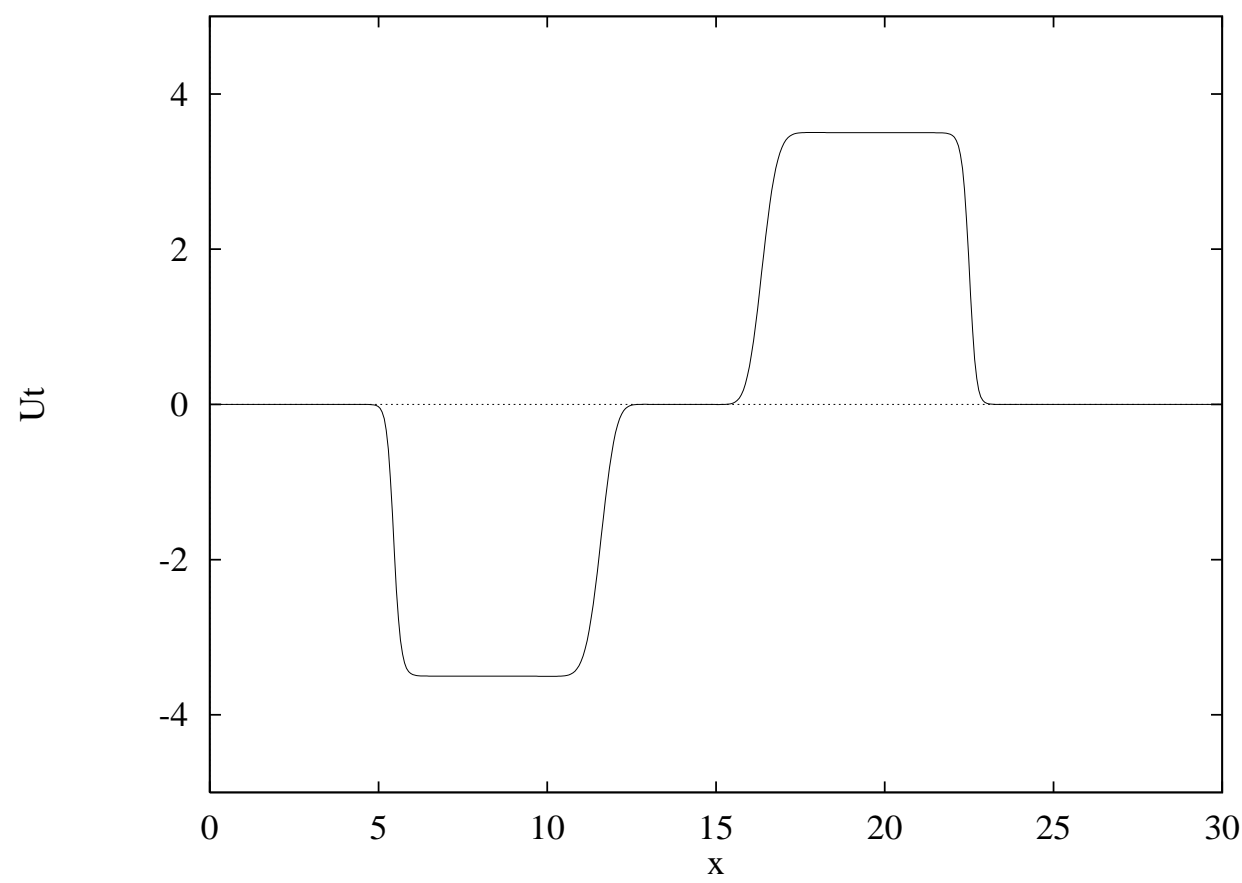

Figure 10: Case 2; distribution of tangential velocity $U_{t}$ obtained with the present scheme (RNC). 


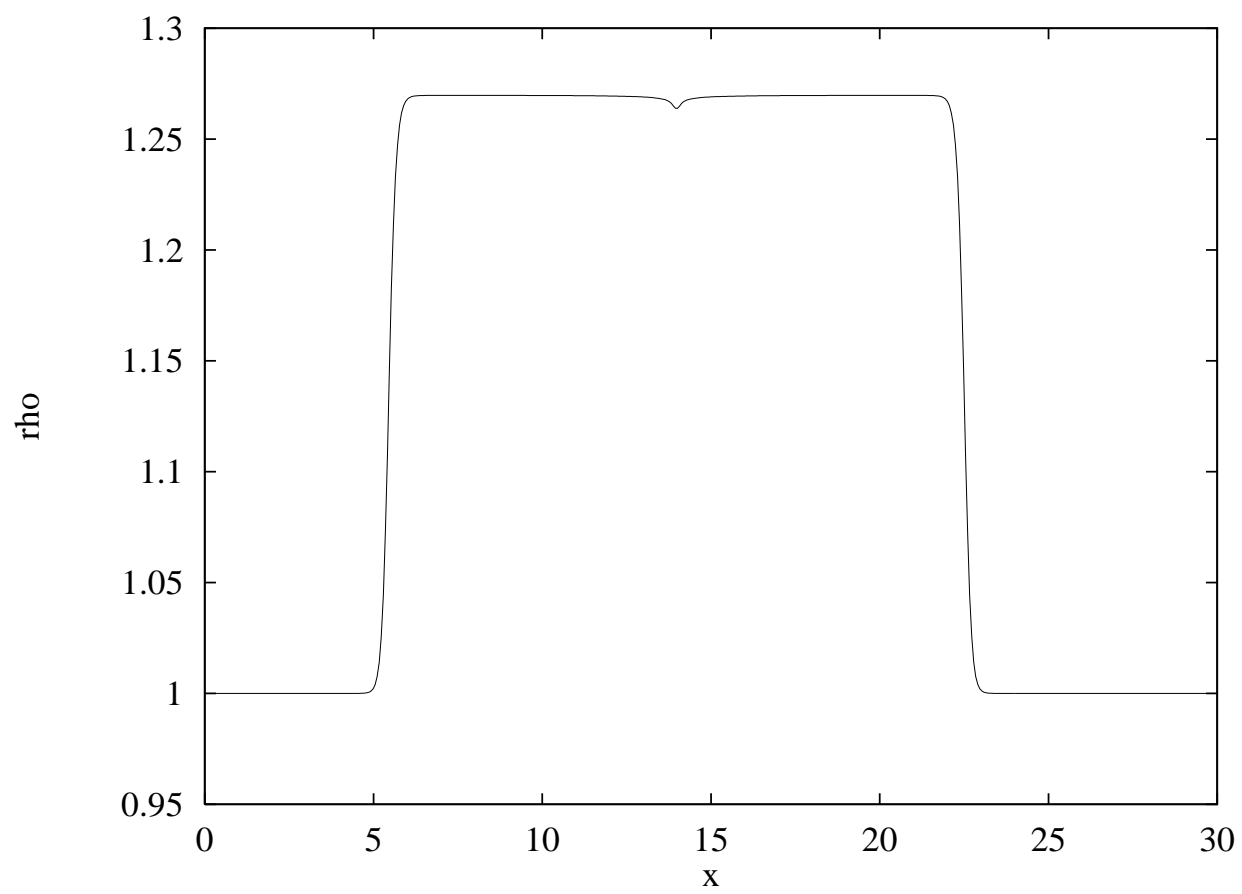

Figure 11: Case 2; distribution of density $\rho$ obtained with the present scheme (RNC).

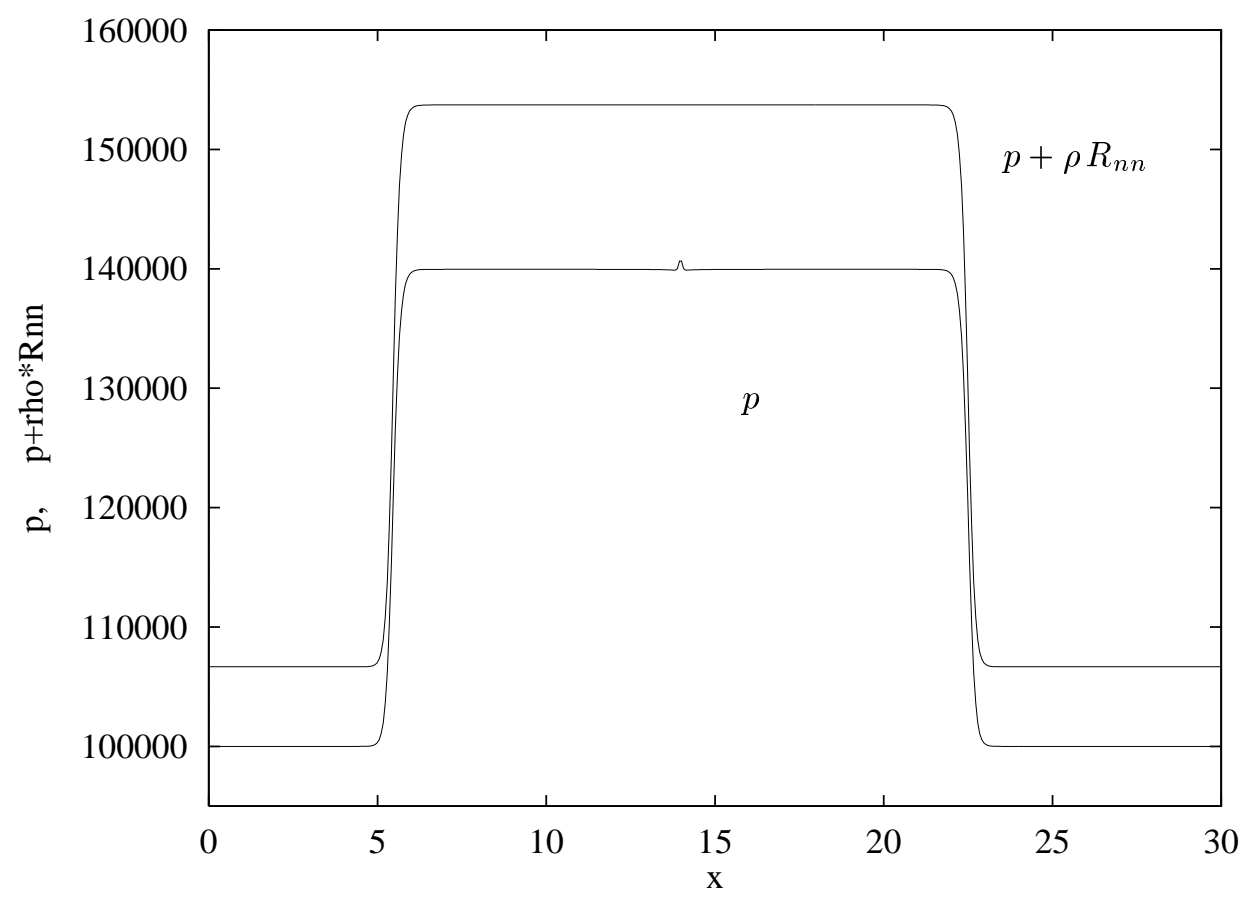

Figure 12: Case 2; distribution of pressure $p$ and total normal stress $p+\rho R_{n n}$ obtained with the present scheme (RNC). 

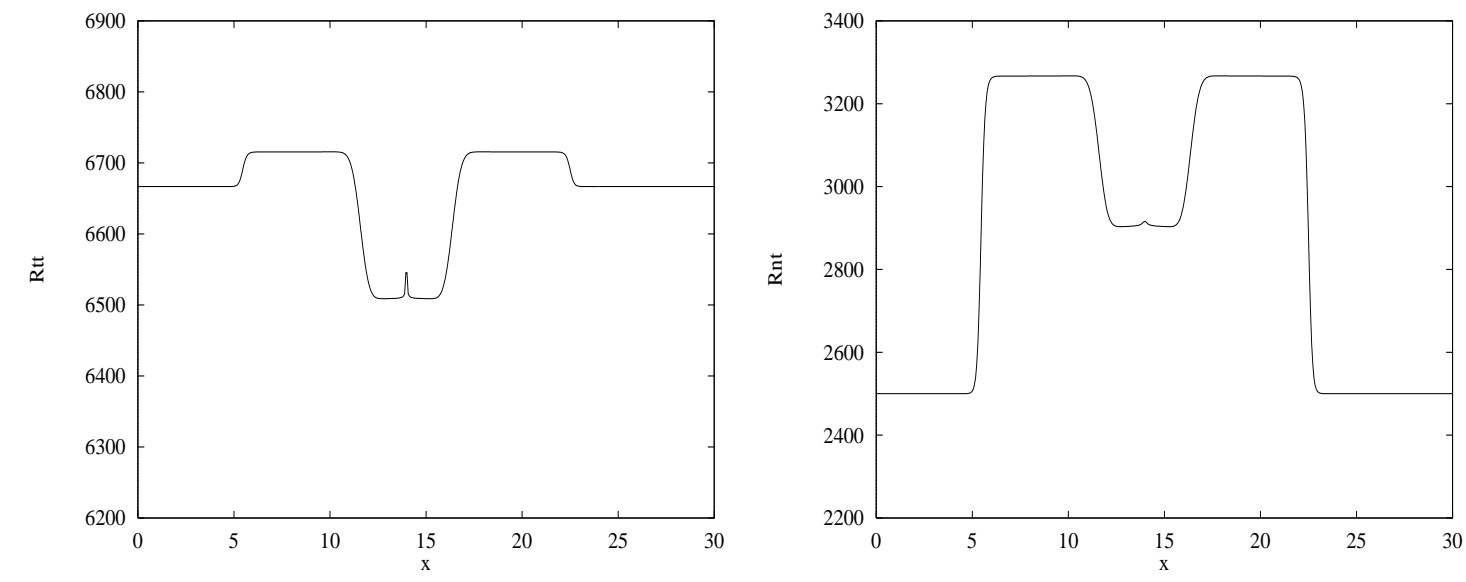

Figure 13: Case 2; distribution of stress components $R_{t t}$ and $R_{n t}$ obtained with the present scheme (RNC).

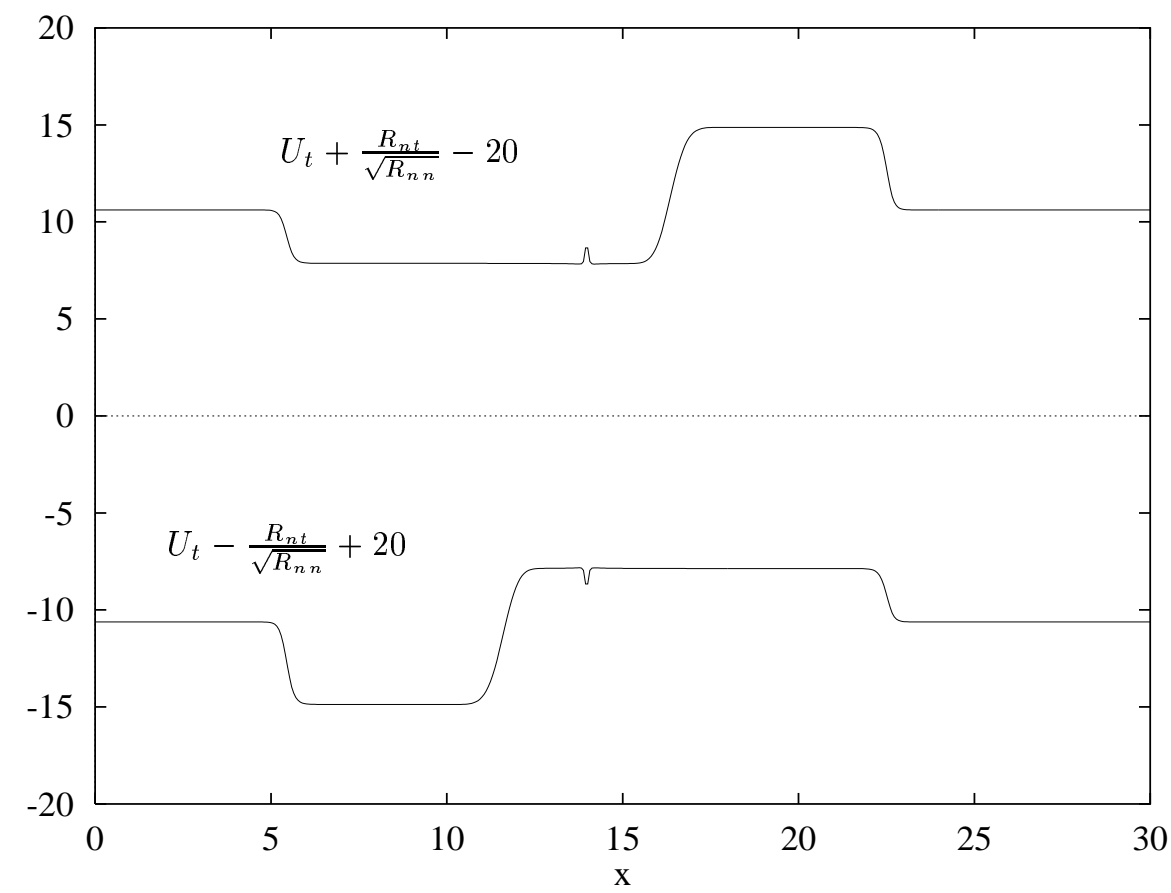

Figure 14: Case 2; distribution of invariants $U_{t} \pm R_{n t} / \sqrt{R_{n n}}$ obtained with the present scheme (RNC). 


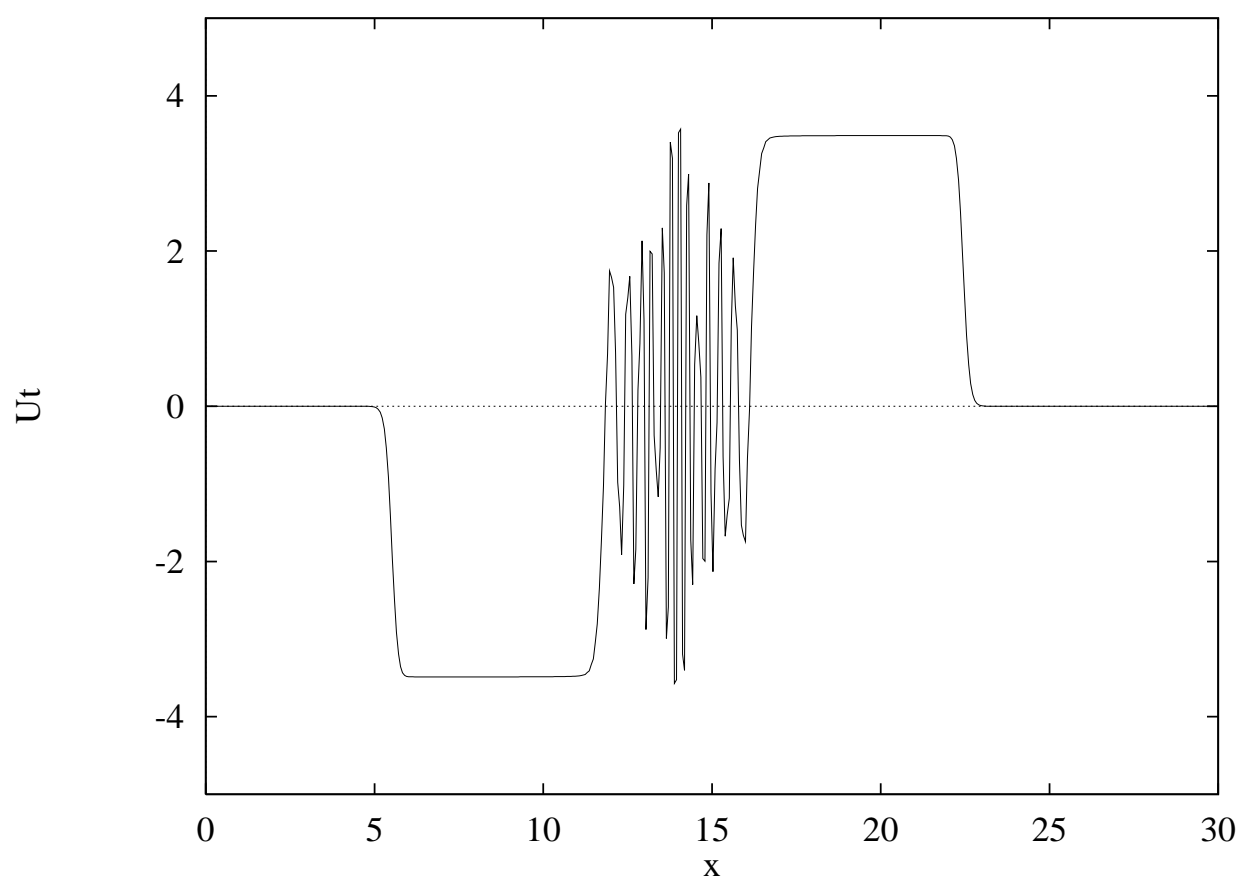

Figure 15: Case 2; distribution of tangential velocity $U_{t}$ obtained with the 'decoupled approach'.

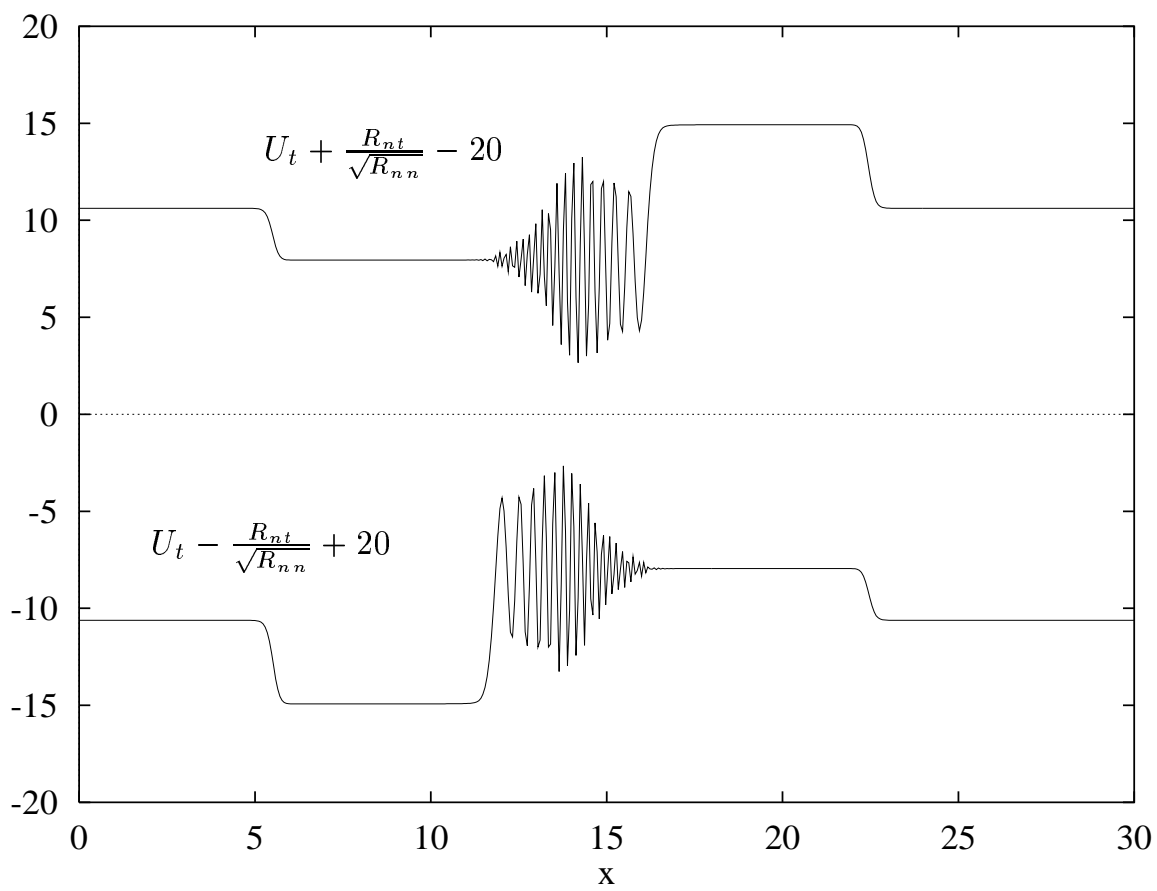

Figure 16: Case 2; distribution of invariants $U_{t} \pm R_{n t} / \sqrt{R_{n n}}$ obtained with the 'decoupled approach'. 


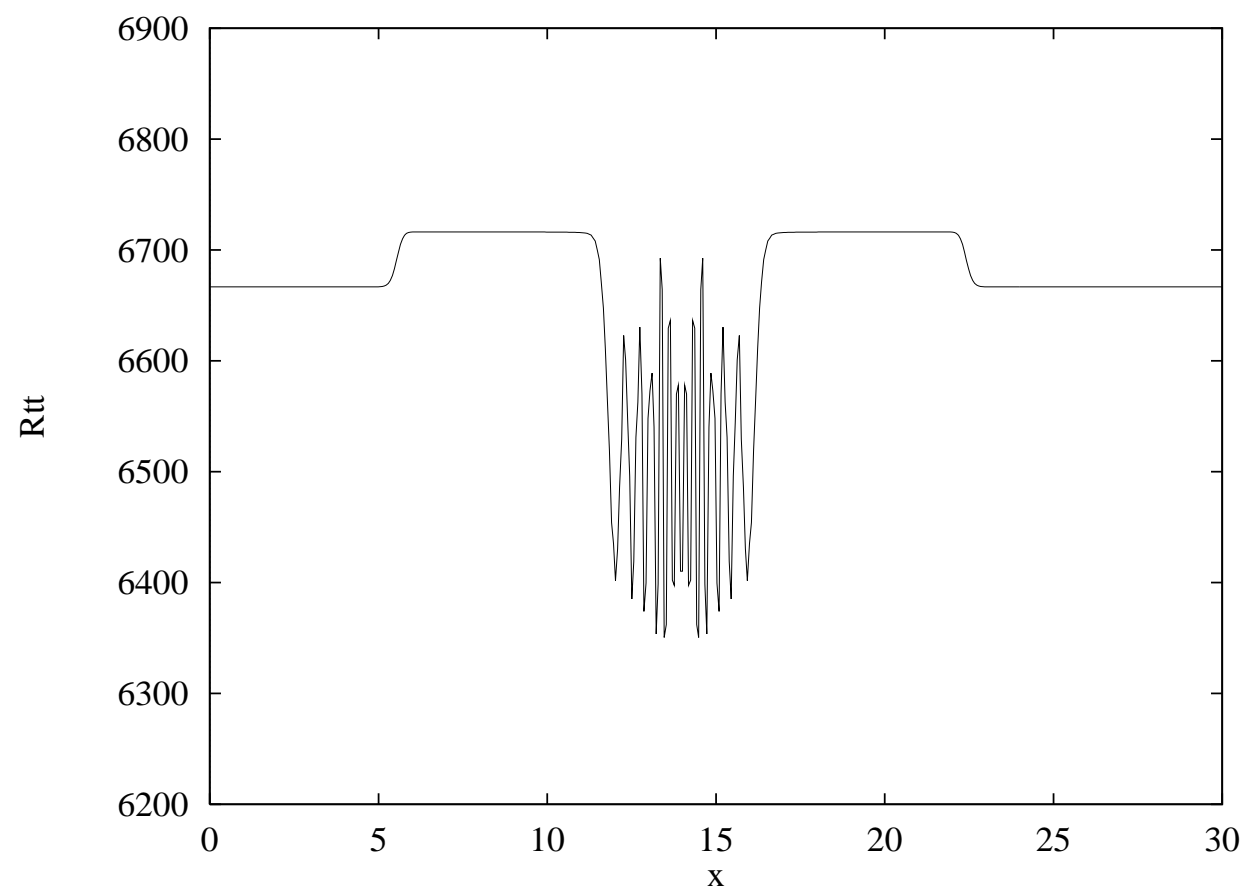

Figure 17: Case 2; distribution of stress component $R_{t t}$ obtained with the 'decoupled approach'.

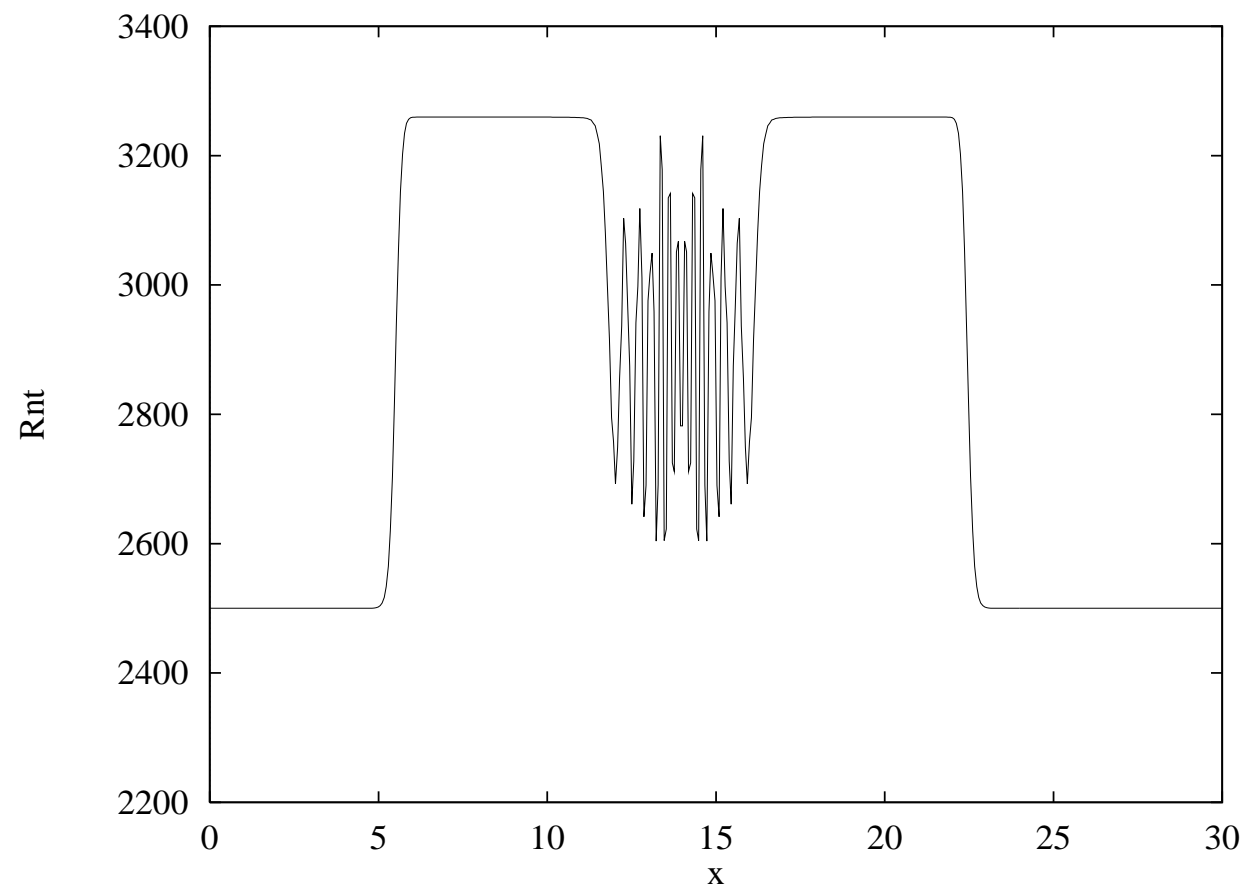

Figure 18: Case 2; distribution of stress component $R_{n t}$ obtained with the 'decoupled approach'. 


\section{A Over-realizability of the Reynolds stress in the hyper- bolic subset}

We wish to verify that the 2 - and 7 -wave do not coincide with the $3-4-5$-6-wave ensuring the existence of a diagonal form of the system of equations (27). For this purpose we examine the Reynolds stress normal component $R_{n n}$, rewriting it as a function of Reynolds stress eigenvalues $\lambda_{(\alpha)}$ and a new unitary vector $n^{\prime}$,

$$
R_{n n}=\mathbf{n}^{\prime} \cdot\left[\begin{array}{cc}
\lambda_{(1)} & 0 \\
0 & \lambda_{(2)}
\end{array}\right] \mathbf{n}^{\prime}=\lambda_{(1)} n_{x}^{2}+\lambda_{(2)} n_{y}^{\prime 2}
$$

Since components $n_{x}^{\prime}$ and $n_{y}^{\prime}$ cannot be zero simultaneously, at least one of the eigenvalues $\lambda_{(\alpha)}$ must vanish in order for $R_{n n}$ to vanish, implying in turn a zero value for the determinant $\delta_{2}^{3}=$ $\lambda_{(1)} \lambda_{(2)}$. Using the conservation law of mass (20), Reynolds stress transport can be expressed as

$$
\left(R_{i j}\right)_{, t}+U_{k}\left(R_{i j}\right)_{, k}+R_{i k}\left(U_{j}\right)_{, k}+R_{j k}\left(U_{i}\right)_{, k}=0
$$

so that we obtain the following evolution equation for the determinant

$$
\left(\delta_{2}^{3}\right)_{, t}+U_{k}\left(\delta_{2}^{3}\right)_{, k}=2 \delta_{2}^{3} U_{k, k}
$$

Integration for regular solutions gives

$$
\delta_{2}^{3}(\mathbf{x}, t)=\delta_{2}^{3}\left(x_{0}, t_{0}\right) \cdot \exp \left(2 \int_{t_{0}}^{t} U_{k_{, k}} \mathrm{~d} t\right),
$$

so that $\delta_{2}^{3}$ cannot vanish over finite intervals if its initial value is non-zero. Hence, over-realisability of the convective subset (20) (positivity of $R_{n n}$ ) is assured for initially over-realizable states.

\section{B Diagonalization of the hyperbolic subset}

It is of practical use to work with the following set of "primitive" variables

$$
\mathbf{P}=\left(\rho, U_{n}, U_{t}, p, R_{n n}, R_{r t t}, R_{s s}, R_{n t}\right)^{t},
$$

transforming system (36) into

$$
\mathbf{P}_{, t}+\tilde{\mathbf{A}} \cdot \mathbf{P}_{, n}=0, \quad \tilde{\mathbf{A}}=\mathbf{M} \cdot \mathbf{A}_{n} \cdot \mathbf{M}^{-1},
$$

where the transformation matrices are

$$
\mathbf{M}=\frac{\partial \mathbf{Z}}{\partial \mathbf{P}}=\left(\begin{array}{cccccccc}
1 & 0 & 0 & 0 & 0 & 0 & 0 & 0 \\
u & \rho & 0 & 0 & 0 & 0 & 0 & 0 \\
v & 0 & \rho & 0 & 0 & 0 & 0 & 0 \\
\frac{u^{2}+v^{2}}{2}+\frac{R_{n n}+R_{t t}+R_{s s}}{2} & \rho u & \rho v & \frac{1}{\gamma-1} & \frac{\rho}{2} & \frac{\rho}{2} & \frac{\rho}{2} & 0 \\
R_{n n} & 0 & 0 & 0 & \rho & 0 & 0 & 0 \\
R_{t t} & 0 & 0 & 0 & 0 & \rho & 0 & 0 \\
R_{s s} & 0 & 0 & 0 & 0 & 0 & \rho & 0 \\
R_{n t} & 0 & 0 & 0 & 0 & 0 & 0 & \rho
\end{array}\right)
$$


and

$$
\mathbf{M}^{-1}=\left(\begin{array}{cccccccc}
1 & 0 & 0 & 0 & 0 & 0 & 0 & 0 \\
-\frac{u}{\rho} & \rho^{-1} & 0 & 0 & 0 & 0 & 0 & 0 \\
-\frac{v}{\rho} & 0 & \rho^{-1} & 0 & 0 & 0 & 0 & 0 \\
\frac{\left(u^{2}+v^{2}\right)(\gamma-1)}{2} & -u(\gamma-1) & -v(\gamma-1) & (\gamma-1) & \frac{(\gamma-1)}{2} & \frac{(\gamma-1)}{2} & \frac{(\gamma-1)}{2} & 0 \\
-\frac{R_{n n}}{\rho} & 0 & 0 & 0 & \rho^{-1} & 0 & 0 & 0 \\
-\frac{R_{t t}}{\rho} & 0 & 0 & 0 & 0 & \rho^{-1} & 0 & 0 \\
-\frac{R_{s s}}{\rho} & 0 & 0 & 0 & 0 & 0 & \rho^{-1} & 0 \\
-\frac{R_{n t}}{\rho} & 0 & 0 & 0 & 0 & 0 & 0 & \rho^{-1}
\end{array}\right) .
$$

The system matrix takes the following simple form

$$
\widetilde{\mathbf{A}}=\left(\begin{array}{cccccccc}
U_{n} & \rho & 0 & 0 & 0 & 0 & 0 & 0 \\
\frac{R_{n n}}{\rho} & U_{n} & 0 & \frac{1}{\rho} & 1 & 0 & 0 & 0 \\
\frac{R_{n t}}{\rho} & 0 & U_{n} & 0 & 0 & 0 & 1 & 0 \\
0 & \gamma p & 0 & U_{n} & 0 & 0 & 0 & 0 \\
0 & 2 R_{n n} & 0 & 0 & U_{n} & 0 & 0 & 0 \\
0 & 0 & 2 R_{n t} & 0 & 0 & U_{n} & 0 & 0 \\
0 & 0 & 0 & 0 & 0 & 0 & U_{n} & 0 \\
0 & R_{n t} & R_{n n} & 0 & 0 & 0 & 0 & U_{n}
\end{array}\right),
$$

allowing for a diagonalization $\tilde{\mathbf{A}}=\mathbf{L} \cdot \Lambda \cdot \mathbf{L}^{-1}$ with

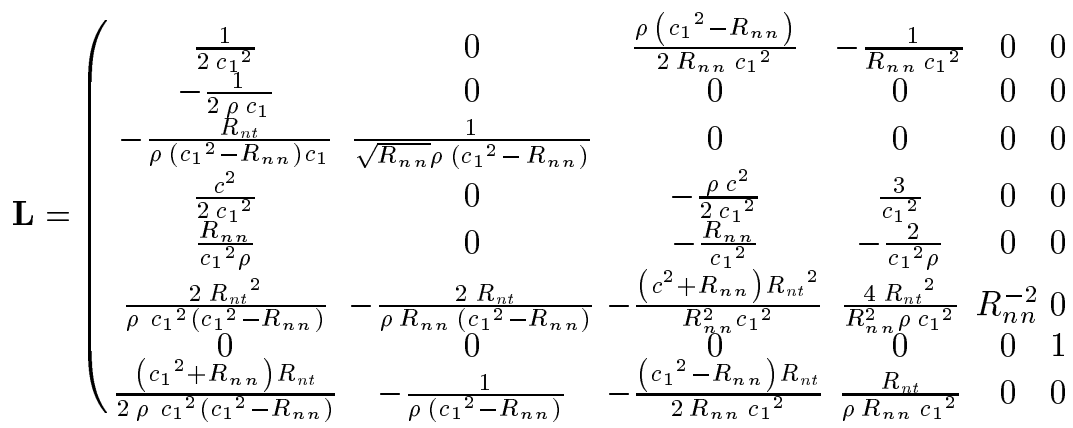

$$
\begin{aligned}
& \left.\begin{array}{cc}
0 & \frac{1}{2 c_{1}^{2}} \\
0 & \frac{1}{2 \rho c_{1}} \\
-\frac{1}{\sqrt{R_{n n}} \rho\left(c_{1}^{2}-R_{n n}\right)} & \frac{R_{n t}}{\rho\left(c_{1}^{2}-R_{n n}\right) c_{1}} \\
0 & \frac{c^{2}}{2 c_{1}^{2}} \\
0 & \frac{R_{n n}}{c_{1}^{2} \rho} \\
-\frac{2 R_{n t}}{\rho R_{n n}\left(c_{1}^{2}-R_{n n}\right)} & \frac{2 R_{n t}{ }^{2}}{\rho c_{1}^{2}\left(c_{1}^{2}-R_{n n}\right)} \\
0 & 0 \\
-\frac{1}{\rho\left(c_{1}^{2}-R_{n n}\right)} & \frac{\left(c_{1}^{2}+R_{n n}\right) R_{n t}}{2 \rho c_{1}^{2}\left(c_{1}^{2}-R_{n n}\right)}
\end{array}\right),
\end{aligned}
$$




$$
\mathbf{L}^{-1}=\left(\begin{array}{cccccccc}
R_{n n} & -c_{1} \rho & 0 & 1 & \rho & 0 & 0 & 0 \\
-\frac{R_{n t} c^{2}}{2}-\sqrt{R_{n n}} R_{n t} \rho & \frac{\sqrt{R_{n n}} \rho\left(c_{1}{ }^{2}-R_{n n}\right)}{2} & R_{n t} \rho R_{n t} & 0 & 0-\frac{\rho\left(c_{1}{ }^{2}-R_{n n}\right)}{2} \\
\frac{2 R_{n n}}{\rho} & 0 & 0 & 0 & -1 & 0 & 0 & 0 \\
0 & 0 & 0 & R_{n n}-\frac{\rho c^{2}}{2} & 0 & 0 & 0 \\
0 & 0 & 0 & 0 & R_{n t}{ }^{2} & R_{n n}^{2} & 0 & -2 R_{n t} R_{n n} \\
0 & 0 & 0 & 0 & 0 & 0 & 1 & 0 \\
-\frac{R_{n t} c^{2}}{2} & \sqrt{R_{n n}} R_{n t} \rho & -\frac{\sqrt{R_{n n}} \rho\left(c_{1}{ }^{2}-R_{n n}\right)}{2} & R_{n t} \rho R_{n t} & 0 & 0-\frac{\rho\left(c_{1}{ }^{2}-R_{n n}\right)}{2} \\
R_{n n} & c_{1} \rho & 0 & 1 & \rho & 0 & 0 & 0
\end{array}\right),
$$

and $\Lambda=\operatorname{diag}\left(\lambda_{1}, \ldots, \lambda_{8}\right)$. Right eigenvectors are simply columns of matrix $\mathbf{L}$, i.e. $r_{k}^{i}=L_{k i}$. Finally, the diagonalization matrices of the original system (36) are obtained through the following relations:

$$
\mathcal{R}=\mathbf{M} \cdot \mathbf{L}, \quad \mathcal{R}^{-1}=\mathbf{L}^{-1} \cdot \mathbf{M}^{-1} .
$$

\section{Derivation of the approximate jump conditions}

Let us consider a system of equations under non-conservative form:

$$
\mathbf{V}_{, t}+\mathbf{D} \cdot \mathbf{V}_{, x}=0
$$

A generalized Rankine-Hugoniot relation has been established by Le Floch [36] in the context of the theory of distributions. This relation is developped in the limit of infinitesimally weak shocks and represents an approximation for non-zero shock strengths. It can be written as:

$$
\int_{0}^{1}\{-\sigma \cdot \mathbf{I}+\mathbf{D}(\phi)\} \frac{\partial \phi}{\partial \xi} \mathrm{d} \xi=0
$$

where $\phi=\phi\left(\xi, \mathbf{V}_{l}, \mathbf{V}_{r}\right)$ represents the path connecting states to the left and to the right of the discontinuity in state-space (see figure 19). Choosing a linear path, i.e. $\phi=[\mathbf{V}] \cdot \xi+\mathbf{V}_{l}$, we obtain

$$
-\sigma[\mathbf{V}]+[\mathbf{V}] \int_{0}^{1} \mathbf{D}(\phi) \mathrm{d} \xi=0
$$

We need to choose a set of variables $\mathbf{V}$ upon which this linear path is imposed. Inspired by the choice of Forestier et al. in the case of a two-equation model [9] we select

$$
\mathbf{V}=\left(\frac{1}{\rho}, U_{n}, U_{t}, p, \rho R_{n n}, \rho R_{t t}, \rho R_{s s}, \rho R_{n t}\right)^{t}
$$

The matrix $\mathbf{D}$ appearing in equation (77) is calculated via the transformation $\mathbf{D}=\mathbf{B}^{-1} \tilde{\mathbf{A B}}$, where $\mathbf{B}=\partial \mathbf{Z} / \partial \mathbf{V}$. Since components $D_{i j}$ are linear functions of components of the vector $\mathbf{V}$, the integration in (77) results in arithmetic means, viz:

$$
-\sigma[\mathbf{V}]+[\mathbf{V}] \cdot \mathbf{D}(\overline{\mathbf{V}})=0 .
$$

Retransformation into our original variables $\mathbf{Z}$ finally yields

$$
-\sigma[\mathbf{Z}]+[\mathbf{F}]+\overline{\mathbf{C}^{n c}}\left(\mathbf{Z}_{l}, \mathbf{Z}_{r}\right)[\mathbf{Z}]=0,
$$



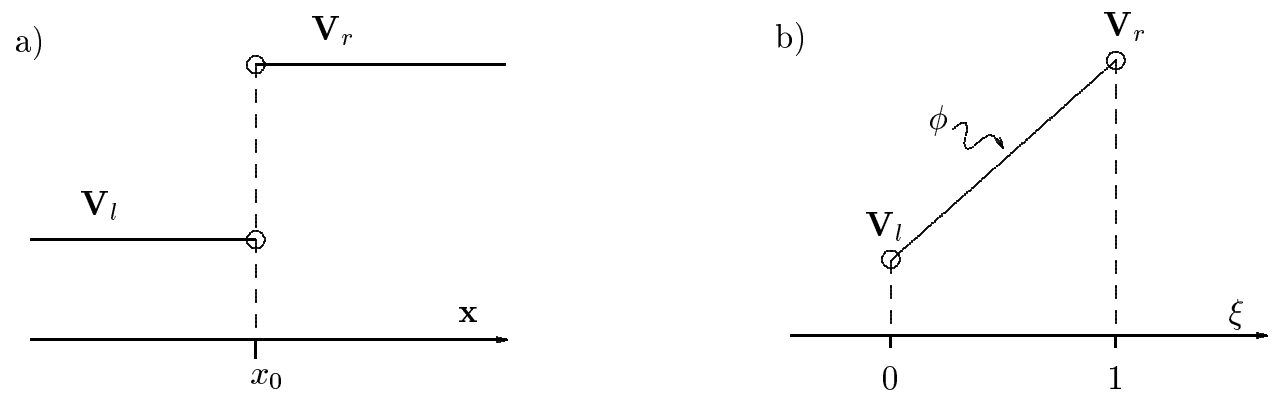

Figure 19: a) Discontinuity of the variable $\mathbf{V}$ in the plane $(\mathbf{x}, \mathbf{V})$. b) Connection of left and right states by a linear path $\phi$ in the plane $(\xi, \mathbf{V})$.

where the matrix $\overline{\mathbf{C}^{n c}}$ contains the following elements:

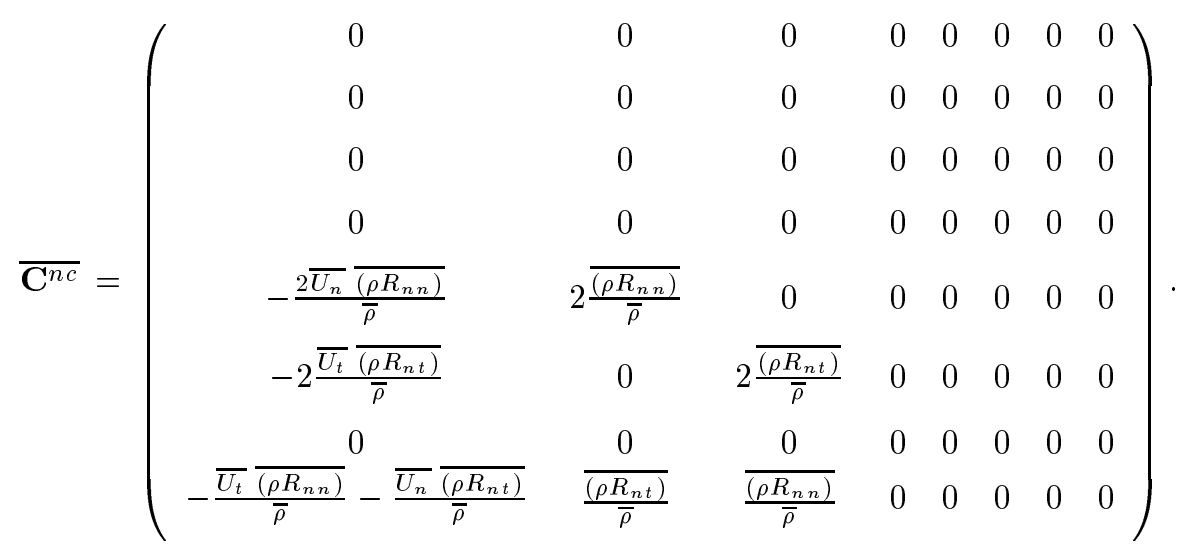

We remark that the above theory based on a linear path fulfils two important conditions of consistency. Firstly, it reduces to the exact Rankine-Hugoniot relations in the limit of zero turbulence, as is obvious from equation (80) and the definition (81). Secondly, it is easily verified that for LD fields the above approximate jump conditions (80) are equivalent to the Riemann invariants of section 3.3.2 which are exact.

\section{Solution to remaining jump conditions}

For the remaining variables of the set, not treated in section 3.3.3, the approximate jump relations across shock waves are the following:

$$
\begin{aligned}
& {\left[U_{t}\right]=} \frac{\left(R_{n t}\right)_{l} \rho_{l}\left[U_{n}\right] \frac{2}{\gamma-1}(z-\beta)}{\rho_{l}\left(R_{n n}\right)_{l} \frac{2}{\gamma-1}(z-\beta)+p_{l} \gamma(z-3)}, \\
& {\left[R_{t t}\right]=} \frac{4(\gamma-1)(z-\beta)\left(R_{n t}\right)_{l}^{2} \rho_{l}}{z\left(\rho_{l}\left(R_{n n}\right)_{l} 2(\gamma-1)(z-\beta)+p_{l} \gamma(z-3)\right)^{2}} \cdot\left\{p_{l}\left(\gamma\left(1-z^{2}\right)+4 z(\gamma-1)\right)\right. \\
&\left.+\rho_{l}\left(R_{n n}\right)_{l} 2(\gamma-1)(z-\beta)(z-1)\left(1+\frac{3(z-1)}{z(2-z)}\right)\right\}, \\
& {\left[R_{s s}\right]=} 0, \quad-\left(R_{n t}\right)_{l} \frac{\rho_{l}\left(R_{n n}\right)_{l} 2(\gamma-1)(z-\beta)\left(\frac{(z-1)(2-z)-3}{z(2-z)}\right)+p_{l} \gamma(z-1)(z+1)}{z\left(\rho_{l}\left(R_{n n}\right)_{l} 2(\gamma-1)(z-\beta)+p_{l} \gamma(z-3)\right)} . \\
& {\left[R_{n t}\right]=}
\end{aligned}
$$




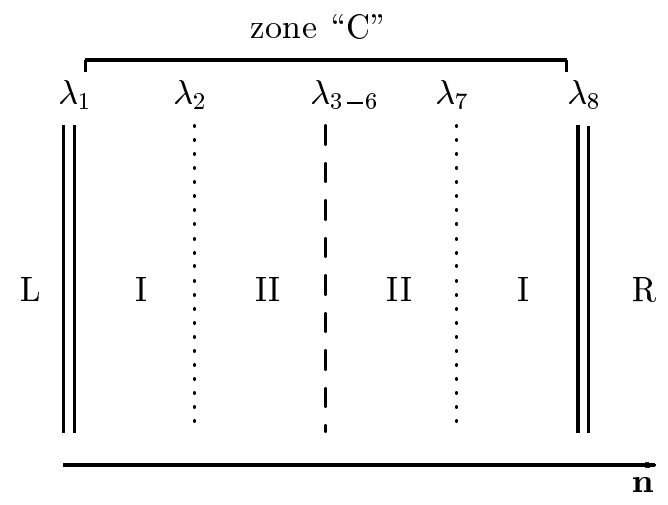

Figure 20: Configuration of the characteristic wave system in the case of a symmetrical double shock. Zone "C" indicates the region where $\left\{\rho, p, R_{n n}, U_{n}\right\}$ are constant and the convective system of equations simplifies considerably.

\section{E Instability of the 'decoupled approach' in the case of a symmetrical problem}

Focussing on case 2 of section 5.3, we note that due to the symmetry of the problem all variables have a zero jump across the centered discontinuity (3-4-5-6-wave) and that the normal velocity $U_{n}$ is zero in the center. Furthermore, the set of variables $\left\{\rho, p, R_{n n}, U_{n}\right\}$ is invariant with respect to the 2- and 7-wave, such that

$$
\rho_{I}=\rho_{I I}, \quad p_{I}=p_{I I}, \quad U_{n I}=U_{n I I}=0, \quad R_{n n I}=R_{n n I I} .
$$

Consequently, between the 1-shock and the 8-shock (zone " $\mathrm{C}$ " in figure 20) the convective system of equations (36) then reduces to the following simple form:

$$
\begin{aligned}
& \left(U_{t}\right)_{, t}+\left(R_{n t}\right)_{, n}=0 \\
& \left(R_{n t}\right)_{, t}+R_{n n}\left(U_{t}\right)_{, n}=0 \\
& \left(R_{t t}\right)_{, t}+2 R_{n t}\left(U_{t}\right)_{, n}=0 .
\end{aligned}
$$

It can be seen that $R_{t t}$ plays a passive role in this zone such that the subsystem consisting of the respective equations for $U_{t}$ and $R_{n t}$ - being linear - will be considered separately in the following.

Since the simple 'decoupled approach' (cf. section 4.2) is based on a characteristic wave system that is essentially equivalent to the Euler waves - not containing waves 2 and 7 of the present system - the numerical flux within zone "C" does not contain an upwind contribution. Applying this explicit central scheme to (87) leads to the following discrete system:

$$
\begin{aligned}
U_{t i}^{n+1}-U_{t_{i}}^{n}+\frac{\Delta t}{2 \Delta x}\left(R_{n t_{i+1}}^{n}-R_{n t_{i-1}^{n}}^{n}\right) & =0 \\
R_{n t_{i}^{n+1}}^{n+}-R_{n t_{i}}^{n}+\frac{\Delta t}{2 \Delta x} R_{n n}\left(U_{t_{i+1}}^{n}-U_{t_{i-1}}^{n}\right) & =0 .
\end{aligned}
$$

The von Neumann analysis (e.g. [43, I,p.296]) of the above system, i.e. inserting a single harmonic

$$
U_{t j}^{p}=\hat{U}_{t}^{p} \cdot e^{I j \phi}, \quad R_{n t}^{p}=\hat{R}_{n t}^{p} \cdot e^{I j \phi},
$$

with $\phi$ being the phase angle and $I=\sqrt{-1}$, we obtain the following system describing the propagation of the error:

$$
\left[\begin{array}{c}
\hat{U}_{t}^{n+1} \\
\hat{R}_{n t}^{n+1}
\end{array}\right]=\underbrace{\left[\begin{array}{cc}
1 & -\frac{\Delta t}{\Delta x} \sin (\phi) \\
-R_{n n} \frac{\Delta t}{\Delta x} \sin (\phi) & 1
\end{array}\right]}_{G(\phi)} \cdot\left[\begin{array}{c}
\hat{U}_{t}^{n} \\
\hat{R}_{n t}^{n}
\end{array}\right] .
$$


The eigenvalues $\lambda_{1,2}$ of the amplification matrix $G(\phi)$,

$$
\lambda_{1,2}=1 \pm I \sin (\phi) \frac{\Delta t}{\Delta x} \sqrt{R_{n n}}
$$

lead to the following expression for the spectral radius $\rho$ :

$$
\rho(G(\phi))=\max _{i=1,2}\left|\lambda_{i}\right|=\sqrt{1+\sin ^{2}(\phi)\left(\frac{\Delta t}{\Delta x}\right)^{2} R_{n n}} \geq 1 .
$$

The 'decoupled approach' is thus susceptible to temporal oscillations and eventual instability for all finite time steps $\Delta t$ and spatial resolutions $\Delta x$. 